\title{
Jenseits von Schwerkraft und Höllenfeuer. Nicht-lineare Kontexteffekte auf den Zusammenhang von religiöser Gruppenzugehörigkeit und individuellem Wahlverhalten bei fünf Bundestagswahlen
}

\author{
Kathrin Ackermann / Richard Traunmüller
}

Beyond Gravity and Hellfire. Non-linear Contextual Effects on the Link between Religion and Voting Behaviour in Five German Federal Elections

Abstract: This article investigates the moderating effect of social context on the relationship between religion and vote choice. Whereas theories of electoral research and the sociology of religion assume a linear contextual effect, we develop a new theoretical argument that predicts a non-linear effect. The results of logistic multilevel analyses confirm this theory for the Catholic milieu: The effect of catholic denomination on voting for the Christian Democrats (CDU/CSU) first increases with the share of Catholics in the context and decreases when they are in the clear majority. This finding - which contradicts former studies - holds for all federal elections under consideration (1994-2009), withstands several robustness tests and provides important evidence for the perpetuation of the traditional Catholic voting norm through group processes.

Keywords: Cleavage-voting, religion, contextual effects, non-linear, federal elections

Schlagwörter: Cleavage-Voting, Religion, Kontexteffekte, Nicht-Linearität, Bundestagswahlen

\section{Einleitung ${ }^{1}$}

Dass Religion einen wahlentscheidenden Faktor darstellen kann, wird von vielen politischen Beobachtern gewürdigt. Die US-amerikanische Debatte dreht sich dabei vor allem um die sogenannten ,culture wars', in denen sich religiöse und säkulare Gruppen in politischen Streitfragen wie Abtreibung, Rechte für Homosexuel-

1 Frühere Versionen dieses Artikels wurden auf der Jahrestagung des „Arbeitskreises Quantitative Religionsforschung“ (AQR) 2012 in Leipzig und auf dem Kongress der „Schweizerischen Vereinigung für Politische Wissenschaft" (SVPW) 2013 in Zürich vorgestellt. Wir danken den Teilnehmern an diesen beiden Veranstaltungen, den anonymen Gutachtern und Herausgebern der PVS sowie insbesondere Marc Bühlmann, Markus Freitag, Simon Munzert, Sigrid Roßteutscher, Peter Selb und Daniel Stegmueller für hilfreiche Hinweise und Kommentare. Der Zweitautor dankt zudem dem „Mannheimer Zentrum für Europäische Sozialforschung“ (MZES), welches ihn während der Fertigstellung des Artikels bestens beherbergt hat. 
le oder Familienwerte gegenüberstehen und welche in einem ausgeprägten ,god gap' bei den jüngeren Präsidentschaftswahlen mündeten (Campbell 2007; Fiorina 2005; Gelman et al. 2008; Green 2010; Hunter 1991; Layman u. Green 2006). In Europa widmet sich ein wiedererwachtes Interesse der Stabilität und dem Wandel traditioneller religiöser Konfliktlinien, welche in der Vergangenheit die Parteienlandschaft vieler europäischer Staaten geprägt haben und das Wahlverhalten breiter Bevölkerungsschichten nach wie vor entscheidend strukturieren (Brooks et al. 2004; Elff 2007; Essmer u. Pettersson 2007; Knutsen 2004; Minkenberg 2010). Neben dem ,neuen', von der Ablösungsthese postulierten religiösen Cleavage zwischen Religiösen und Nicht-Religiösen scheinen hier auch die ,alten“ konfessionellen Konfliktlinien Bestand zu haben (Roßteutscher 2012; Wolf 1996). Debatten über die abnehmende Relevanz sozialstruktureller Merkmale für politisches Verhalten sowie Modernisierungs- und Säkularisierungsthesen zum Trotz, konnte auch für die jüngsten Wahlen in Deutschland nachgewiesen werden, dass Religionszugehörigkeit das Wahlverhalten beeinflusst: Katholiken haben nach wie vor eine höhere Wahrscheinlichkeit, die Unionsparteien zu wählen, als alle anderen (Arzheimer u. Schoen 2007; Brooks et al. 2004; Debus 2010; Elff u. Roßteutscher 2011; Jagodzinski u. Quandt 2000; Jung et al. 2010; Klein u. Pötschke 2000; Lachat 2007; Minkenberg 2010; Pappi u. Brandenburg 2010; Raymond 2011; Roßteutscher 2007, 2012; Schoen 2009; van der Brug et al. 2009; Weßels 2011).

Während der Zusammenhang zwischen Konfession und Wahlentscheidung auf Individualebene hinreichend untersucht wurde, wissen wir nur sehr wenig über die sozialen Prozesse, welche diese Beziehung aufrechterhalten oder Religion überhaupt erst politisch salient werden lassen (Brettschneider et al. 2002). Ein sozialstrukturelles Merkmal wie die Konfessionszugehörigkeit ist per se von geringer politischer Relevanz. Für cleavage-voting sind vielmehr der soziale Kontext und der Kontakt mit Mitgliedern der eigenen sozialen Gruppe von entscheidender Bedeutung (Berelson et al. 1954; Levine 2005). Dies erkennt sowohl die Wahlforschung als auch die Religionssoziologie an: „The political ramification of religious adherence are a by-product of regularly placing oneself in a social milieu where politically relevant messages are conveyed indirectly but regularly, often through casual conversation" (Olson 2012, S. 105). Mit anderen Worten ist der Einfluss von Religion auf politisches Verhalten nicht als Individualeffekt zu begreifen, sondern hängt entscheidend vom sozialen Kontext ab.

In diesem Beitrag gehen wir der Frage nach, welchen Effekt die konfessionelle Komposition des sozialen Kontextes auf die Beziehung zwischen religiöser Gruppenzugehörigkeit und individueller Wahlentscheidung bei Bundestagswahlen hat. Theoretisch kann man dabei auf zwei - zum einen aus der Wahlforschung und zum anderen aus der Religionssoziologie stammende - Argumente zurückgreifen, welche beide zur selben Vorhersage gelangen: Die Stärke des Einflusses religiöser Gruppenzugehörigkeit auf das Wahlverhalten wird durch die Größe der Religionsgruppe innerhalb eines Kontextes moderiert. Laut der wahlsoziologischen These vom Gesetz der sozialen Schwerkraft wird ein Mitglied einer sozialen Gruppe umso eher die diese Gruppe repräsentierende Partei wählen, je höher ihr Anteil im sozialen Kontext ist (Tingsten [1937] 1975). In gleicher Weise postuliert die religionssoziologische Höllenfeuer-Hypothese, dass ein Individuum religiöse 
Normen umso stärker befolgt, je eher sie in seinem sozialen Kontext geteilt und wirksam sanktioniert werden (Stark u. Bainbrigde 1996).

Die theoretische Innovation unserer Studie besteht in der Entwicklung eines neuen theoretischen Arguments zum Kontexteffekt auf die politische Salienz von Religion. Während sowohl das Gesetz der sozialen Schwerkraft als auch die Höllenfeuer-Hypothese von einem linearen Kontexteffekt ausgehen - je höher der Anteil der eigenen Gruppe im Kontext, desto stärker der Individualeffekt der Konfession - argumentieren wir für eine nicht-lineare funktionale Form des Zusammenhangs von sozialem Kontext und dem Einfluss individueller Religionszughörigkeit. Bei dem sozialen Prozess, welcher diesen moderierenden Kontexteffekt steuert, gehen wir von einer besonderen Bedeutung der konfessionellen Gegengruppe als Objekt der identitätsstiftenden Abgrenzung und des politischen Wettbewerbs aus. Ist eine starke Gegengruppe vorhanden, entstehen Anreize zur sozialen Abgrenzung und politischen Auseinandersetzung. Dies mündet in eine identitätsstiftende Wirkung des konfessionellen Merkmals und in eine höhere politische Salienz von Religion. Entsprechend sollte, je nach Grad der religiösen Polarisierung im Kontext, der moderierende Effekt der eigenen Konfessionsgruppe unterschiedlich stark ausfallen. Kurz gesprochen hängt die politische Salienz von Religion unserer These zufolge vom konfessionellen Wettbewerb im sozialen Umfeld ab, wobei wir einen tipping point des Moderatoreffekts erwarten: Während der Effekt der individuellen Konfession auf die Wahlentscheidung mit zunehmendem Katholikenanteil zunächst ansteigt, sollte er wieder schwächer werden, sobald die Katholiken in einer eindeutigen Mehrheitsposition sind.

Empirisch leisten wir mit unserer Studie einen Beitrag zur Kontroverse um Kontexteffekte auf das politische Verhalten in Deutschland (Arzheimer 2009; Dülmer u. Ohr 2008; Klein 2007; Klein u. Pötschke 2000; Pickery 2002; van Deth u. Tausendpfund 2013). Jenseits der allgemeinen Frage, ob soziale Kontexte politisches Verhalten überhaupt nennenswert strukturieren, konnte der spezifische moderierende Kontexteffekt des katholischen Sozialmilieus zuletzt nur noch bei der Bundestagswahl 1969 nachgewiesen werden, was als Ende der politischen Prägekraft des sozialen Kontextes interpretiert wurde (Klein 2007; Klein u. Pötschke 2000). Unsere Analysen widersprechen diesem Befund. Wir testen unser Argument empirisch für die fünf Bundestagswahlen 1994, 1998, 2002, 2005 und 2009, wobei jeweils die 326 westdeutschen Kreise und kreisfreien Städte als Kontexteinheiten fungieren. Die Ergebnisse logistischer Mehrebenenanalysen bestätigen eindeutig einen nicht-linearen Kontexteffekt für das katholische Sozialmilieu: Der Effekt der katholischen Konfession auf die Wahrscheinlichkeit einer Wahl der Unionsparteien steigt für Katholiken bei zunehmendem Katholikenanteil im Kontext zunächst an und nimmt wieder ab, sobald die Katholiken eindeutig in der Mehrheit sind. Dieses Ergebnis findet sich für alle fünf betrachteten Bundestagswahlen, hält Robustheitsanalysen stand und ist damit ein wichtiges Indiz dafür, dass die traditionelle katholische Wahlnorm nach wie vor in sozialen Interaktionsprozessen aufrechterhalten wird.

Schließlich haben unsere Befunde auch wichtige Implikationen für die in der Religionssoziologie lebhaft geführte Debatte zwischen Anhängern der klassischen Säkularisierungstheorie (Berger 1990; Bruce 2002; Pickel 2010) und Vertretern 
des religionsökonomischen Marktmodells (Iannaccone 1991; Stark u. Finke 2000). Die empirischen Ergebnisse stützen die religionsökonomische Vorstellung von der mobilisierenden Wirkung des konfessionellen Wettbewerbs und widersprechen dem säkularisierungstheoretischen Argument, wonach religiöse Konkurrenz mit einem sozialen Bedeutungsverlust der Religion einhergeht.

Die vorliegende Studie ist wie folgt aufgebaut. Zunächst entwickeln wir unser theoretisches Argument und erläutern die Annahmen sowie die daraus abgeleiteten Propositionen und Hypothesen. Im Anschluss daran werden die verwendete Methode sowie die Datenbasis und Messung der theoretischen Konzepte dargestellt. Darauf folgen die Präsentation der empirischen Ergebnisse sowie eine Reihe an Robustheitsanalysen. Der Artikel schliesst mit einem Fazit.

\section{Theorie}

Individuen treffen Entscheidungen über ihr politisches Verhalten nicht isoliert, sondern werden durch ihre Umwelt beeinflusst: „People develop their political preferences, knowledge, values, perceptions of ability, and decisions about political behavior in interaction with others, usually members of their social circles" (Zuckerman 2007, S. 635; vgl. auch Przeworski u. Soares 1971). Hinsichtlich des für das politische Verhalten relevanten sozialen Umfelds kann - basierend auf den frühen Werken der Columbia School - zwischen einer engen Kontextdefinition im Sinne von sozialen Netzwerken (Baldassarri 2009; Levine 2005; McClurg 2003; Mutz 2002a, 2002b; Schmitt-Beck et al. 2012; Verba et al. 2005; Zuckerman et al. 2007) und einer stärker geografisch geleiteten Definition des sozialen Kontextes (Books u. Prysby 1991; Bühlmann 2006; Bühlmann u. Freitag 2006; Dyck et al. 2009; Gimpel et al. 2004; Gimpel u. Lay 2005; Huckfeldt 1979, 1980; Johnston u. Pattie 2006; Klein u. Pötschke 2000; Knack u. Kropf 1998; Pickery 2002) differenziert werden.

Während Netzwerke das soziale Umfeld einer Person bezeichnen, welches auf freiwillig gewählten Interaktionen basiert, stellen Kontexte vielmehr von außen vorgegebene, oft geografisch definierte Umwelten dar. Diese sind der Kontrolle von Individuen weitestgehend entzogen. Das heißt nicht, dass sich Individuen nicht bewusst etwa für ihren Wohnort oder ihre Nachbarschaft entscheiden können. Doch sobald sich eine Person innerhalb eines wie auch immer definierten Kontextes bewegt, wird sie externen Einflüssen ausgesetzt und mit sozialen Interaktionen konfrontiert, die auf sie einwirken, ohne dass diese von ihr bewusst gewählt werden (Carmines u. Huckfeldt 1998; Huckfeldt 2009; Huckfeldt u. Sprague 1987, 1993). Die Dichotomie zwischen Netzwerk und Kontext findet sich auch in der Religionssoziologie. Hier geht der Netzwerkansatz davon aus, dass vor allem Kirchen und religiöse Gemeinden die Grenzen religiöser Umwelten beschreiben. Vertreter des Kontextansatzes argumentieren hingegen, dass diese Grenzen keineswegs so eng zu fassen sind, sondern sich religiös definierte soziale Kontexte weit jenseits der engeren Kirchengemeinde erstrecken (Kotler-Berkowitz 2001; Lenski 1961; Traunmüller 2011, 2012). Die vorliegende Studie verwendet eine geografische Definition und begreift den Landkreis bzw. die kreisfreie Stadt als relevanten sozialen Kontext einer Person. 
Bevor konkrete Propositionen und Hypothesen bezüglich des Kontexteffekts abgeleitet werden, werden zunächst notwendige Annahmen formuliert. Diese werden nicht getestet, sondern als gegeben betrachtet. Die erste Annahme geht davon aus, dass der weite soziale Kontext die Interaktionen eines Individuums strukturiert. Eine Person ist in ihren alltäglichen Interaktionen auf die Personen beschränkt, denen sie im Kontext begegnet. Wen eine Person an der Arbeitsstelle, im Verein, in der Kirchengemeinde, in der Kneipe, im Supermarkt oder auf dem Schulweg der Kinder trifft, wird durch die Zusammensetzung des Kontextes bestimmt. Interaktionen werden folglich durch die Komposition des weiten sozialen Umfelds strukturiert (Blau 1977; Blau u. Schwartz 1984; Burbank 1997; Huckfeldt 1983, 2007; Huckfeldt u. Sprague 1993).

Je mehr Mitglieder der eigenen sozialen Gruppe im Kontext anwesend sind, desto größer ist die Chance, ihnen in den eben beschriebenen Alltagssituationen $\mathrm{zu}$ begegnen. Daher wird außerdem angenommen, dass eine steigende relative Gruppengröße die Interaktionswahrscheinlichkeit mit Angehörigen der sozialen Gruppe erhöht. Wenngleich Menschen ohnehin dazu neigen, eher mit Angehörigen der eigenen sozialen Gruppe zu interagieren, so wird diese ingroup-Tendenz durch die Gruppengröße noch einmal verstärkt (Blau 1977; Blau u. Schwartz 1984, S. 30-40)

Es wird weiterhin die Annahme getroffen, dass durch die lokale Interaktion mit Angehörigen der eigenen, durch ein sozialstrukturelles Merkmal definierten Gruppe dieses Merkmal überhaupt erst politisch salient wird. Für sich allein genommen übt ein sozialstrukturelles Merkmal keine Wirkung aus: „Social categories serve analytical purposes and they can be on occasion good proxies for social groups, but it is from the web of local interactions that characterize people's social groups that social cleavages and political identities are produced and reinforced" (Baldassarri 2009, S. 4). Erst die Interaktion mit Mitgliedern der eigenen Gruppe führt zur Entwicklung einer Identität, welche wiederum politisch salient werden kann: „Der Einzelne hat eine Identität nur im Bezug zu den Identitäten anderer Mitglieder seiner gesellschaftlichen Gruppe“ (Mead 1973, S. 206). Durch die Entwicklung einer auf die soziale Gruppe bezogenen Identität verhält sich ein Individuum auch politisch entsprechend den Normen dieser Gruppe.

\subsection{Schwerkraft und Höllenfeuer - Kontexteffekte in der Wahl- und Religionssoziologie}

Mit diesen Annahmen gehen wir zusammenfassend davon aus, dass der soziale Kontext Interaktionen strukturiert, die Interaktionswahrscheinlichkeit mit den Angehörigen der eigenen sozialen Gruppe mit der Größe dieser Gruppe im Kontext steigt und die politische Wirksamkeit eines sozialstrukturellen Merkmals der Interaktion mit Angehörigen der eigenen sozialen Gruppe bedarf. Führt man diese Annahmen zusammen, lässt sich daraus folgende Proposition ableiten: Die politische Salienz eines individuellen sozialstrukturellen Merkmals nimmt mit der Stärke der eigenen sozialen Gruppe im Kontext zu. Die Wahrscheinlichkeit einer Interaktion mit Angehörigen der eigenen sozialen Gruppe ist dann höher und das 
Merkmal kann durch diese Interaktionen seine politische Bedeutung entfalten. Diese Steigerung der politischen Wirksamkeit des Merkmals durch das soziale Umfeld wird als Moderatoreffekt des Kontextes auf den Zusammenhang zwischen dem Individualmerkmal und politischem Verhalten verstanden.

Sowohl in der Wahlforschung als auch in der Religionssoziologie ist diese These zu finden. Die wahlsoziologische Variante geht zurück auf das von Tingsten ([1937] 1975, S. 230) formulierte Gesetz der sozialen Schwerkraft: „The attachment of a particular group to the party which on the whole may be regarded as representing the group most closely, rises with the strength of the group within the area. For instance, the workers vote more consistently socialist in proletarian than in more well-to-do districts." Aus religionssoziologischer Perspektive lässt sich die Höllenfeuer-Hypothese von Stark und Bainbrigde (1996, S. 72) anführen: "What counts is not only whether a particular person is religious, but whether this religiousness is or is not ratified by the social environment. The idea here is that religion is empowered to produce conformity to the norms only as it is sustained through interaction and accepted by the majority as a valid basis for action”. Beide Thesen sprechen von einer Verstärkung der sozialen Identität und den damit verbundenen Normen - seien es Wahlnormen oder religiöse Normen - durch die Präsenz der eigenen sozialen Gruppe, welche diese Normen vorgibt, unterstützt und wirksam sanktioniert.

Für die hier betrachteten Katholiken sollte folglich die politische Wirksamkeit der Konfession mit zunehmendem Katholikenanteil im Kontext an Stärke gewinnen. Vor dem historischen Hintergrund des katholischen Sozialmilieus in Deutschland (Liedhegener 2006) und im Anschluss an die Befunde der Wahlforschung auf individueller Ebene, spricht dies für eine Verstärkung der Wahlnorm für die Unionsparteien. Es lässt sich daher folgende Hypothese formulieren:

Hypothese 1: Der Effekt der katholischen Konfessionszugehörigkeit auf die individuelle Wahlwahrscheinlichkeit der Unionsparteien nimmt mit steigendem Katholikenanteil im Landkreis bzw. der kreisfreien Stadt zu.

\subsection{Jenseits von Schwerkraft und Höllenfeuer - nicht-lineare Kontexteffekte}

Hypothese 1 beschreibt einen linear verlaufenden Kontexteffekt des Katholikenanteils auf den Individualzusammenhang zwischen Konfession und Wahlverhalten: Mehr Katholiken im Kontext erhöhen die Stärke des Individualzusammenhangs. Der Effekt wäre dann bei einem hypothetischen Katholikenanteil von 100 Prozent am höchsten. Wenn dieser Kontexteffekt über die Betonung der eigenen sozialen Identität und den damit verbundenen politischen Normen verläuft, bedarf es jedoch einer weiteren Qualifizierung hinsichtlich der getroffenen Annahmen.

Die Formierung einer auf einem sozialstrukturellen Merkmal basierenden und auf eine soziale Gruppe bezogenen Identität kann nur dann erfolgen, wenn sich die Angehörigen einer Gruppe auch tatsächlich durch eben dieses Merkmal von anderen unterscheiden können: „[...] by definition ingroups necessarily imply outgroups [...]“ (Brewer 1999, S. 432). Erst wenn dies gegeben ist, entfaltet das 
sozialstrukturelle Merkmal seine identitätsstiftende Wirkung. Es wird daher zusätzlich angenommen, dass für die Entwicklung einer sozialen Gruppenidentität die Abgrenzung zu anderen Gruppen notwendig ist.

Konkret bedeutet dies, dass die politische Identität von Katholiken durch Interaktionen mit anderen Angehörigen des katholischen Milieus geprägt und verstärkt wird, die sich in ihren politischen Vorstellungen und Präferenzen wiederum von „Nicht-Katholiken“ unterscheiden. In diesem Sinne bietet das katholische Milieu vor allem durch Abgrenzung von Anderen ein Umfeld zur wirksamen Entwicklung eines politischen Selbstverständnisses. Der Schlüssel zur politischen Wirksamkeit der katholischen Identität ist daher das Vorhandensein einer sichtbaren nicht-katholischen Gegengruppe (Bearman u. Brückner 2001; Geser 1997). Die Gruppengröße und damit die Stärke dieser Gegengruppe verhalten sich invers zum Anteil der Katholiken im Kontext und werden folglich von ihm bestimmt. ${ }^{2}$

Führt man die erste Proposition und die formulierte Qualifikation zusammen, ergibt sich eine zweite Proposition. Demnach nimmt die politische Salienz eines sozialstrukturellen Merkmals mit steigender Gruppengröße zunächst zu und ab einer gewissen Gruppenstärke wieder ab.

Diese Proposition lässt sich anhand von drei Szenarien verdeutlichen. Sind die Katholiken in der deutlichen Minderheit, fehlt die Möglichkeit zur Interaktion, wodurch das sozialstrukturelle Merkmal politisch nicht salient werden kann. Der erwartete Einfluss des Individualmerkmals auf politisches Verhalten ist daher zunächst gering. Wenn die konfessionelle Komposition des Kontexts durch ein ungefähres Gleichgewicht beider Gruppen geprägt ist und somit die Polarisierung und der konfessionelle Wettbewerb am höchsten sind, sollte die gegenseitige Abgrenzung am besten möglich sein. Entsprechend erreicht das sozialstrukturelle Merkmal in diesem Kontext die maximale politische Salienz. Diese Argumentation wird durch die empirischen Befunde von van der Brug et al. (2009) gestützt, die in ihrer Untersuchung von Europawahlen zu dem Ergebnis kommen, dass Religion die Wahlentscheidung vor allem in religiös fraktionalisierten Kontexten beeinflusst. ${ }^{3}$ Sie argumentieren, dass in diesen Kontexten eine Politisierung religiöser Verhaltensweisen und Gefühle stattfinde und Religion dadurch politisch relevant werde. Sobald jedoch die eigene Gruppe im Kontext so stark vertreten ist, dass andere Gruppen kaum noch sichtbar sind - wenn der Katholikenanteil beispielsweise knapp 100 Prozent erreicht - ist die soziale Abgrenzung, welche für die Entwicklung der Gruppenidentität notwendig ist, nicht mehr möglich. In diesem Fall sollte der politische Einfluss des Individualmerkmals abnehmen, da es dann keine identitätsstiftende Wirkung mehr entfaltet. Dies entspricht dem Argu-

2 Um eine klare Dichotomie zwischen zwei konfessionellen Gruppen zu erreichen, namentlich zwischen Katholiken und Protestanten, werden die nachfolgenden empirischen Analysen auf Westdeutschland beschränkt. Die religiöse Landschaft in Ostdeutschland ist aufgrund des hohen Anteils der Konfessionslosen diverser und folgt nicht der beschriebenen Dichotomie zwischen den Katholiken einerseits und den Protestanten als Gegengruppe andererseits (Roßteutscher 2012).

3 In unserer Studie wäre eine hohe Fraktionalisierung gleichbedeutend mit einer hohen Polarisierung, da wir von einer klaren Dichotomie zwischen Katholiken und Protestanten in Westdeutschland ausgehen. Bei zwei Gruppen stehen Fraktionalisierung und Polarisierung in einem perfekten linearen Zusammenhang. 
ment, dass gerade in stark katholisch geprägten Kontexten der Anreiz, eine spezifische katholische Kultur zu entwickeln, fehlt (Geser 1997). Es lässt sich daher eine Hypothese ableiten, die auf einen umgekehrt u-förmigen Moderatoreffekt verweist:

Hypothese 2: Der Effekt der katholischen Konfessionszugehörigkeit auf die individuelle Wahlwahrscheinlichkeit der Unionsparteien steigt mit zunehmendem Katholikenanteil im Landkreis bzw. der kreisfreien Stadt zunächst an und nimmt wieder ab, sobald Katholiken in der deutlichen Mehrheit sind.

Konzeptionell sagt Hypothese 2 damit einen sogenannten tipping point voraus (Huckfeldt 1980). Der kontextuelle Anteil einer sozialen Gruppe kann theoretisch einen kritischen Schwellenwert erreichen, ab dem sich die damit verbundenen sozialen Prozesse und ihre Folgen - etwa hinsichtlich ihrer politischen Bedeutung verändern. Formal gesprochen bedeutet ein tipping point eine Diskontinuität in den Werten einer Variablen in Abhängigkeit der Werte einer zweiten Variablen. Ob der tipping point und damit der stärkste Einfluss der Konfession auf die Wahlentscheidung tatsächlich bei einem Katholikenanteil von 50 Prozent liegt, wie es das Argument der religiösen Polarisierung vorhersagen würde, oder ob der Einfluss des Individualmerkmals erst dann abnimmt, wenn die Katholiken in der wie auch immer zu quantifizierenden überwältigenden Mehrheit sind, ist eine empirische Frage, welcher wir uns im Folgenden zuwenden.

\section{Statistische Modellierung}

Die statistische Modellierung stellt das Bindeglied zwischen der entwickelten Theorie und den empirischen Daten dar. In Hypothese 1 und 2 werden statistische Zusammenhänge postuliert, welche anhand eines logistischen varying intercept, vaying slope Modells mit Cross-Level-Interaktion getestet werden können (Gelman u. Hill 2007):

$$
\begin{gathered}
\operatorname{Pr}\left(y_{i j}=1\right)=\operatorname{logit}{ }^{(-1)}\left(a_{j}+\beta_{j} x_{i j}\right), \text { für } \mathrm{i}(=1, \ldots, \mathrm{N}) \text { Befragte, } \\
\alpha_{j}=\mu_{\alpha}+\gamma_{\alpha} z_{j}+\eta_{\alpha j}, \text { für } \mathrm{j}(=1, \ldots, \mathrm{J}) \text { Kreise, } \\
\beta_{j}=\mu_{\beta}+\gamma_{\beta} z_{j}+\eta_{\beta j}, \text { für } \mathrm{j}(=1, \ldots, \mathrm{J}) \text { Kreise, mit } \\
\eta_{\alpha j} \sim N\left(0, \sigma_{\alpha}\right) \\
\eta_{\beta j} \sim N\left(0, \sigma_{\beta}\right) \\
\operatorname{cor}\left(\eta_{\alpha j}, \eta_{\beta j}\right)=\rho .
\end{gathered}
$$

Der Katholikenanteil $z_{j}$ im Landkreis bzw. in der kreisfreien Stadt $j$ stellt in diesem Fall das Kontextmerkmal dar, welches laut der Hypothesen auf den Individualzusammenhang zwischen Konfession $\chi_{i j}$ und der Wahlentscheidung $y_{i j}$ wirkt. Anders ausgedrückt erhält der Effekt der individuellen Konfession $\beta_{j}$ ein eigenes Modell auf Kontextebene. Setzt man die Gleichungen auf Kreisebene in die Gleichung auf Individualebene ein, erhält man:

$$
\operatorname{Pr}\left(y_{i j}=1\right)=\operatorname{logit}(-1)\left(\mu_{\alpha}+\mu_{\beta} \chi_{i j}+\gamma_{\alpha} z_{j}+\gamma_{\beta} z_{j} \chi_{i j}+\eta_{\alpha j}+\eta_{\beta j} \chi_{i j}\right),
$$


wobei der Einfluss der individuellen Konfessionszugehörigkeit nun leicht erkennbar eine Funktion des Kontextmerkmals darstellt, wie der multiplikative Interaktionsterm $\gamma_{\beta} z_{i} x_{i j}$ verdeutlicht. Während diese Modellspezifikation den in Hypothese 1 postulierten linearen Moderatoreffekt abbildet, wird Hypothese 2 zum quadratischen Effekt mit folgender Spezifikation getestet:

$$
\begin{aligned}
& \alpha_{j}=\mu_{\alpha}+\gamma_{1 \alpha} z_{j}+\gamma_{2 \alpha} z_{j}^{2}+\eta_{\alpha j}, \\
& \beta_{j}=\mu_{\beta}+\gamma_{1 \beta} z_{j}+\gamma_{2 \beta} z_{j}^{2}+\eta_{\beta j},
\end{aligned}
$$

beziehungsweise in kompakter Schreibweise:

$$
\operatorname{Pr}\left(y_{i j}=1\right)=\operatorname{logit}(-1)\left(\mu_{\alpha}+\mu_{\beta} x_{i j}+\gamma_{1 \alpha} z_{j}+\gamma_{2 \alpha} z_{j}^{2}+\gamma_{1 \beta} z_{j} x_{i j}+\gamma_{2 \beta} z_{j}^{2} \chi_{i j}+\eta_{\alpha j}+\eta_{\beta j} \chi_{i j}\right),
$$

Wir schätzen getrennte Mehrebenenmodelle für die fünf Bundestagswahlen von 1994 bis 2009 um die zeitliche Stabilität des moderierenden Kontexteffekts zu prüfen. Das verwendete Schätzverfahren basiert auf Maximum Penalized Likelihood, was sich äquivalent zu einer Bayesianischen Schätzung mit Priori-Verteilungen für die unbekannten Varianzkomponenten verhält (Chung et al. 2012). Dazu verwenden wir das blme-package der Statistiksoftware R (Dorie 2011).

\section{Daten}

Zur Durchführung der vorgestellten Mehrebenenanalysen werden die Daten des Forsa-Bus 1995 bis 2010 verwendet. Der Datensatz beruht auf telefonischen Befragungen der „forsa Gesellschaft für Sozialforschung und statistische Analyse“, welche mittels eines kurzen Fragebogens auch politische Einstellungen und politisches Verhalten erfassen. An jedem Werktag werden hierfür ca. N = 500 Personen in Deutschland telefonisch interviewt, sodass pro Jahr Daten für über $\mathrm{N}=$ 120.000 Bürgerinnen und Bürgern zur Verfügung stehen. Aufgrund der hohen Befragtenzahl ist eine Aggregation von Individualmerkmalen auf Ebene der Landkreise bzw. kreisfreien Städte möglich. Wie beschrieben werden für die fünf Bundestagswahlen, welche zwischen 1994 und 2009 stattfanden, jeweils Querschnittanalysen durchgeführt. Dafür werden die jährlich erhobenen Datensätze des Forsa-Bus zu fünf Datensätzen zusammengefügt, wobei der Wahltag jeweils den Stichtag darstellt. ${ }^{4}$

Da wir dabei über längere Zeiträume aggregieren, ignorieren wir natürlich mögliche kurzfristige Veränderungen zwischen den konfessionellen Variablen und dem berichteten Wahlverhalten. Obwohl Schoen (2003) in Anlehnung an Gelman und King (1993) keine systematische zeitliche Variation zwischen individueller Konfession und Wahlabsicht beobachtet, kann Traunmüller (2013) zeigen, dass der Einfluss des katholischen Kontextes auf die Wahlpräferenzen von Katholiken

42005 fand die Bundestagswahl beispielsweise in der 38. Kalenderwoche statt. Folglich werden die Daten, welche vor der 38. Kalenderwoche 2005 erhoben wurden, dem Datensatz für die Bundestagswahl 2002 und die Daten, welche ab der 38. Kalenderwoche 2005 erhoben wurden, dem Datensatz der Bundestagswahl 2005 zugeordnet. 
tatsächlich einer strukturierten Dynamik folgt. Allerdings variiert der Kontexteffekt vor allem mittelfristig von einer Wahl zur nächsten und weniger kurzfristig innerhalb einer Legislaturperiode oder eines Wahlkampfs. Dies ermutigt uns zu der Annahme, dass unsere Ergebnisse durch die gewählte zeitliche Aggregation nicht nennenswert verzerrt werden.

Wir beschränken unsere Analyse auf die J = 326 westdeutschen Kreise und kreisfreien Städte. Dafür gibt es im Wesentlichen zwei Gründe. Erstens wurden in Ostdeutschland seit der Wiedervereinigung eine Reihe von Kreisreformen durchgeführt. Indem wir die Analyse auf Westdeutschland beschränken, können die Kontexteinheiten über Zeit konstant gehalten werden. Zweitens ist die Dichotomie zwischen Katholiken und Nicht-Katholiken im Westen klarer definiert als im Osten. Sie lässt sich hier mehr oder weniger als Nullsummenspiel zwischen Katholiken und Protestanten beschreiben, während sich in Ostdeutschland aufgrund des hohen Anteils an Konfessionslosen ein komplexeres Bild zeigt (Roßteutscher 2012). Die Korrelation zwischen dem Katholiken- und Protestantenanteil (ohne Freikirchen) in westdeutschen Kreisen liegt laut unserer Stichprobe 1994 bei $-0.97,1998$ bei $-0.96,2002$ bei $-0.94,2005$ bei -0.93 und 2009 immerhin noch bei -0.92 .

Die abhängige Variable der vorliegenden Studie - die individuelle Wablentscheidung - wird aus der retrospektiven Frage nach der Wahlentscheidung bei der letzten Bundestagswahl gewonnen. Da das Interesse auf der Wahlwahrscheinlichkeit der Unionsparteien liegt, wird die abhängige Variable dichotom kodiert, sodass eine 1 für eine Wahlentscheidung zu Gunsten von CDU oder CSU und eine 0 für die Wahl einer der übrigen Parteien oder eine Wahlenthaltung steht. In unserer Stichprobe geben stets ein Drittel der Wahlberechtigten an, für eine der beiden Unionsparteien gestimmt zu haben: 199437 Prozent, 199833 Prozent, 200234 Prozent, 200535 Prozent und 200933 Prozent (siehe Tabelle 6 im Anhang). ${ }^{5}$ Abbildung 1 zeigt für die fünf betrachteten Bundestagswahlen zudem die Verteilung der Wähleranteile für CDU und CSU über die Landkreise und kreisfreien Städte hinweg. Es zeigt sich eine deutliche regionale Varianz, wobei - wie zu erwarten - die höchsten Anteile in den Bayrischen Landkreisen und Teilen Nordrhein-Westfalens zu finden sind. 1994 variiert der Wähleranteil für die Unionsparteien zwischen 20 und 55 Prozent, 1998 zwischen 15 und 53 Prozent, 2002 zwischen 18 und 59 Prozent, 2005 zwischen 21 und 52 sowie 2009 zwischen 20 und 55 Prozent.

5 Diese Wähleranteile liegen zum Teil deutlich über den offiziellen Wahlergebnissen für die Wahlberechtigten in Westdeutschland (1994: 31.3, 1998: 27.7, 2002: 28.7, 2005: 25.7 und 2009: 22.5), was auf Umfrageeffekte, z. B. overreporting oder Selektionseffekte, hindeutet. Tatsächlich besteht ein Problem darin, dass wir mit den vorliegenden Daten das Wahlverhalten der Befragten nur über eine retrospektive Frage erheben können. Dies ist insofern problematisch, als sich Befragte möglicherweise nicht richtig an ihr tatsächliches Wahlverhalten erinnern können oder wollen und daher falsche Angaben machen (Schoen 2011). Dieses Problem verschärft sich noch, je weiter eine Wahl in der Vergangenheit liegt. Wir können es hier leider nicht lösen, allerdings legen die Ergebnisse in Traunmüller (2013) nahe, dass es keine zeitlichen Variationen in dem uns hier primär interessierenden Kontexteffekt gibt, welche systematisch mit der verstrichenen Zeit nach der Wahl und damit möglichen Erinnerungsschwierigkeiten zusammenhängen würden. 


\section{Abbildung 1: Anteile der CDU/CSU-Wähler pro Landkreis/kreisfreie Stadt}
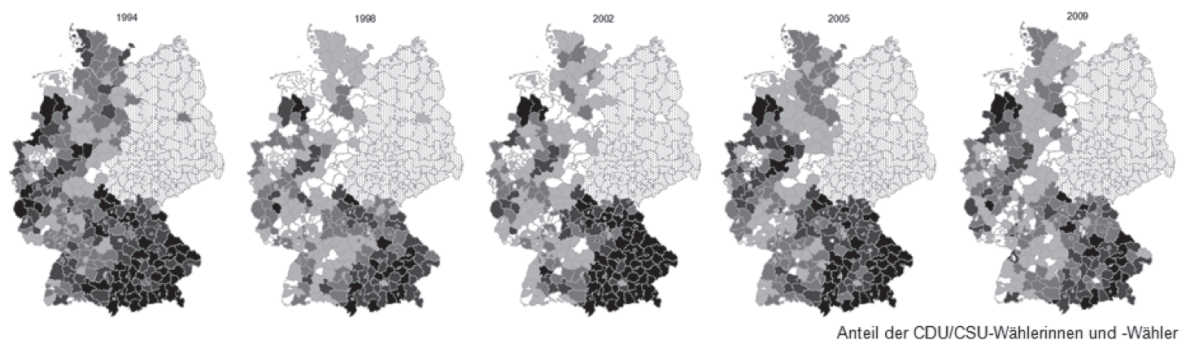

$:$ nicht in der Analyse
$<31$ Prozent
$31-35$ Prozent
$36-40$ Prozent
$41-45$ Prozent
$>45$ Prozent

Quelle: eigene Darstellung auf Basis der Daten des Forsa-Bus 1995-2010

Die primär interessierende erklärende Variable auf Individualebene ist die individuelle Konfession. Da mit den Katholiken eine Religionsgruppe im besonderen Fokus dieser Studie steht, wird auch diese Variable dichotom kodiert. Sie nimmt dann den Wert 1 an, wenn eine befragte Person der römisch-katholischen Kirche formal angehört, und den Wert 0, wenn die Person Mitglied einer anderen oder keiner Religionsgemeinschaft ist. Unsere Daten zeigen für den Beobachtungszeitraum einen leichten Rückgang in den Mitgliedschaften. So sind 199441 Prozent der Befragten katholisch, 199840 Prozent, 200237 Prozent, 200536 Prozent und 2009 nur noch 35 Prozent (siehe Tabelle 6 im Anhang).

Theoretisch relevant ist in dem vorliegenden Papier vor allem der Einfluss der konfessionellen Komposition des Kontextes auf die Individualbeziehung zwischen katholischer Konfession und Wahlentscheidung. Daten zur konfessionellen Struktur auf Ebene der Landkreise und kreisfreien Städte stellt in Deutschland weder die öffentliche noch die kirchliche Statistik zur Verfügung. Deshalb wird zur Messung der interessierenden Variable auf Kontextebene - Katholikenanteil im Landkreis/in der kreisfreien Stadt - die zuvor beschriebene dichotome Individualvariable katholische Konfession herangezogen und auf Ebene des Landkreises bzw. der kreisfreien Stadt aggregiert (zu diesem Vorgehen siehe Dülmer 2005 sowie Dülmer u. Ohr 2008). Dadurch entsteht eine kontinuierliche Variable, welche den Katholikenanteil im Kontext wiedergibt. Die Aggregation erfolgt jeweils pro untersuchter Wahl, um mögliche Veränderungen der konfessionellen Komposition über Zeit zu erfassen. Abbildung 2 stellt den Katholikenanteil je Landkreis bzw. kreisfreie Stadt für die fünf Zeitpunkte dar. Auch hier zeigen sich die bekannten regionalen Unterschiede. Der Katholikenanteil variiert 1994 zwischen 4 und 89 Prozent, 1998 zwischen 4 und 90 Prozent, 2002 zwischen 4 und 88 Prozent, 2005 zwischen 5 und 84 Prozent und schließlich 2009 zwischen 3 und 87 Prozent. 


\section{Abbildung 2: Katholikenanteil pro Landkreis/kreisfreie Stadt}
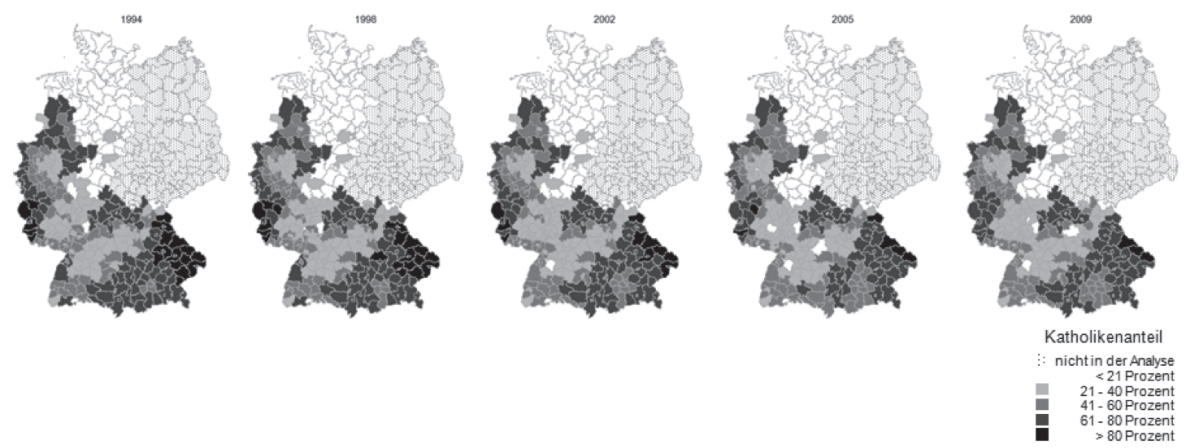

Quelle: eigene Darstellung auf Basis der Daten des Forsa-Bus 1995-2010

Um die Robustheit der Ergebnisse zu testen, werden in späteren Modellen verschiedene Kontrollvariablen berücksichtigt, welche den Individualzusammenhang bzw. den Moderatoreffekt möglicherweise konfundieren. Die genaue Operationalisierung aller Variablen, deren Quellen sowie deskriptive Statistiken finden sich in den Tabellen 5 und 6 im Anhang.

\section{Ergebnisse}

Die empirische Überprüfung unserer Hypothesen erfolgt in mehreren Schritten. In einem ersten Schritt werden vereinfachte Mehrebenenmodelle geschätzt, welche den individuellen Katholikeneffekt über die verschiedenen Kreise variieren lassen, diese Variation jedoch noch nicht modellieren. Stattdessen wird die Beziehung zwischen individuellem Katholikeneffekt und kontextuellem Katholikenanteil zunächst grafisch exploriert, um einen ersten Eindruck über die funktionale Form dieses Zusammenhangs zu erhalten. In einem zweiten Schritt werden lineare und quadratische Spezifikationen des moderierenden Kontexteffekts geschätzt und in ihrer Anpassung an die Daten kontrastiert. Diese beiden funktionalen Formen lassen sich aus Hypothese 1 und 2 ableiten. Im dritten Abschnitt wird dann eine Reihe von Robustheitsanalysen durchgeführt, um zu überprüfen, inwieweit unsere Befunde auch bei alternativen Modellspezifikationen Bestand haben.

\subsection{Exploration}

Tabelle 1 stellt die Ergebnisse der für die fünf Bundestagswahlen getrennt berechneten Mehrebenenmodelle ohne Gleichung für den varying slope dar. Wie erwartet geht die individuelle katholische Konfessionszugehörigkeit zu allen Zeitpunkten mit einer höheren Wahlwahrscheinlichkeit der Unionsparteien einher. Die Größe der Koeffizienten bleibt dabei über den gesamten betrachteten Zeitraum konstant (0.62 bis 0.65 ), wobei die Wahrscheinlichkeit, die Unionsparteien zu 
wählen, bei Katholiken im Durchschnitt stets etwa 15 Prozent höher liegt als bei Nicht-Katholiken. ${ }^{6}$

Tabelle 1: Die Wahlentscheidung für die Unionsparteien bei Katholiken - Basismodell ohne Modellierung des random slopes

\begin{tabular}{|c|c|c|c|c|c|c|c|c|c|}
\hline & \multicolumn{2}{|c|}{1994} & \multicolumn{2}{|c|}{1998} & \multicolumn{2}{|c|}{2002} & \multicolumn{2}{|c|}{2005} & 2009 \\
\hline Konstante & -0.83 & $(0.03)$ & -1.08 & $(0.03)$ & -1.10 & $(0.03)$ & -0.94 & $(0.03)$ & $-0.97 \quad(0.03)$ \\
\hline Katholik & 0.64 & $(0.01)$ & 0.64 & $(0.01)$ & 0.62 & $(0.01)$ & 0.63 & $(0.01)$ & $0.65 \quad(0.02)$ \\
\hline Katholikenanteil & 0.14 & $(0.05)$ & 0.24 & $(0.05)$ & 0.58 & $(0.07)$ & 0.31 & $(0.06)$ & $0.14 \quad(0.06)$ \\
\hline SD Konstante & 0.23 & & 0.25 & & 0.30 & & 0.23 & & 0.20 \\
\hline SD Katholik & 0.17 & & 0.17 & & 0.16 & & 0.14 & & 0.14 \\
\hline Korrelation & -0.58 & & -0.68 & & -0.46 & & -0.52 & & -0.42 \\
\hline $\mathrm{N}$ Individuen & 311 & 230 & 285 & 598 & 262 & 2185 & 341 & 462 & 114425 \\
\hline J Kreise & 32 & 26 & 3. & 26 & & 26 & & 26 & 326 \\
\hline
\end{tabular}

Anmerkung: eigene Berechnung auf Basis des Forsa-Bus 1995-2010; unstandardisierte Koeffizienten (logits) aus logistischen Mehrebenenmodellen; Standardfehler in Klammern.

Entscheidend für die vorliegende Studie ist jedoch, dass die Größe des Katholikeneffekts über die 326 westdeutschen Kreise hinweg variiert. Die Standardabweichung der varying slopes liegt zwischen 0.14 und 0.17. Für die Bundestagswahl 2009 bedeutet dies, dass der Koeffizient für 95 Prozent aller Kreise zwischen 0.37 und 0.93 liegt bzw. die Wahlwahrscheinlichkeit der Unionsparteien für Katholiken je nach Kontext zwischen rund 9 und 23 Prozent höher liegt als für Nicht-Katholiken. Dies sind durchaus beträchtliche kontextuelle Unterschiede in der politischen Wirksamkeit der individuellen Konfessionszugehörigkeit.

Unabhängig von der eigenen Konfessionszugehörigkeit beeinflusst aber auch ein katholisch geprägtes Umfeld die Wahlentscheidung zugunsten der Unionsparteien. Bei allen fünf Bundestagswahlen zeigt sich ein statistisch signifikanter Effekt des regionalen Katholikenanteils, welcher jedoch über die Zeit variiert. Am schwächsten fällt er für die Bundestagswahlen 1994 und 2009 aus (jeweils 0.14), am stärksten zur dazwischenliegenden Bundestagswahl 2002 (0.58). Die substanzielle Größe dieses Effekts ist allerdings eher gering. 2002 etwa schlug sich eine Differenz im Katholikenanteil von 10 Prozent lediglich in einer erhöhten Wahlwahrscheinlichkeit von etwa 1.45 Prozent für die Unionsparteien nieder.

Um einen ersten Eindruck darüber zu erhalten, inwieweit die aufgezeigte Variation der politischen Wirksamkeit der individuellen Konfessionszugehörigkeit mit der konfessionellen Zusammensetzung des sozialen Kontextes zusammenhängt, werden diese beiden Größen in Abbildung 3 in fünf Streudiagrammen zueinander in Beziehung gesetzt. Um neben der Richtung des Zusammenhangs auch dessen funktionale Form besser einschätzen zu können, wird die grafische Darstellung

6 Differenzen in der Wahrscheinlichkeit lassen sich approximieren, indem man die logits durch vier teilt: „As a rule of convenience, we can take logistic regression coefficients (other than the constant term) and divide them by 4 to get an upper bound of the predictive difference corresponding

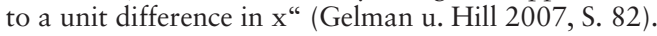


durch eine nicht-parametrische Regressionslinie (loess) unterstützt, welche die Punktewolke zusammenfasst und dadurch bestehende Muster sichtbar macht (Cleveland 1979).

\section{Abbildung 3: Individualeffekt der katholischen Konfession auf die Wahlentschei- dung für die Unionsparteien (shrinkage estimates) bei variierendem Katholiken- anteil im Kontext (grafische Exploration zu Tabelle 1)}
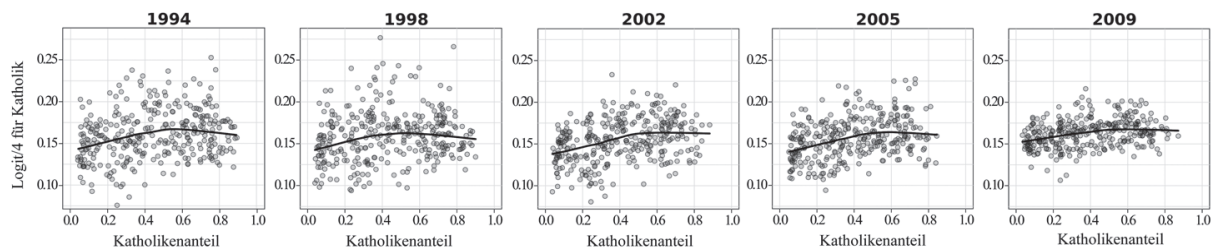

Quelle: eigene Darstellung auf Basis der in Tabelle 1 dargestellten Schätzungen

Für alle fünf Bundestagswahlen ergibt sich ein sehr ähnliches Bild für den Zusammenhang zwischen dem individuellen Katholikeneffekt und dem katholischen Bevölkerungsanteil im Kreis. Der individuelle Katholikeneffekt steigt dabei zunächst mit zunehmendem Katholikenanteil jeweils an und erreicht bei etwa 50 Prozent Katholiken im Kreis seinen Höhepunkt. Bei weiterer Erhöhung des Katholikenanteils nimmt dieser Effekt der individuellen Konfessionszugehörigkeit jedoch wieder ab. Die politische Wirksamkeit des eigenen religiösen Merkmals steht damit in einem systematischen Zusammenhang mit der religiösen Zusammensetzung des sozialen Umfelds. Die explorative Analyse legt weiterhin nahe, dass es sich dabei um einen nicht-linearen, quadratischen Zusammenhang handelt, wie von Hypothese 2 vorhergesagt. Im Folgenden soll diese Beziehung formal getestet werden.

\subsection{Formaler Test}

Tabelle 2 zeigt die Koeffizienten und Standardfehler der logistischen Mehrebenenmodelle mit modellierten varying slopes. Dabei werden zwei alternative Modellspezifikationen getestet - eine mit linearer Cross-Level-Interaktion, welche den in Hypothese 1 formulierten Zusammenhang abbildet, sowie eine zweite mit quadratischem Interaktionsterm, welcher der Aussage von Hypothese 2 entspricht. 


\section{Tabelle 2: Kontexteffekte auf die Wahlentscheidung bei Katholiken - Zwei Modellspezifikationen im Vergleich}

\begin{tabular}{|c|c|c|c|c|c|c|c|c|c|}
\hline \multirow[b]{2}{*}{ Linear } & \multicolumn{2}{|c|}{1994} & \multicolumn{2}{|c|}{1998} & \multicolumn{2}{|c|}{2002} & \multicolumn{2}{|c|}{2005} & \multirow[t]{2}{*}{2009} \\
\hline & & & & & & & & & \\
\hline Katholik & 0.52 & $(0.03)$ & 0.55 & $(0.03)$ & 0.42 & $(0.03)$ & 0.45 & $(0.03)$ & $0.43(0.04)$ \\
\hline Katholikenanteil & -0.03 & $(0.06)$ & 0.10 & $(0.06)$ & 0.31 & $(0.08)$ & 0.06 & $(0.07)$ & $-0.12(0.07)$ \\
\hline Katholik * Katholikenanteil & 0.28 & $(0.06)$ & 0.21 & $(0.06)$ & 0.48 & $(0.06)$ & 0.43 & $(0.06)$ & $0.55(0.08)$ \\
\hline \multicolumn{10}{|l|}{ Quadratisch } \\
\hline Katholik & 0.45 & $(0.05)$ & 0.46 & $(0.05)$ & 0.35 & $(0.05)$ & 0.37 & $(0.05)$ & $0.31(0.07)$ \\
\hline Katholikenanteil & -1.25 & $(0.23)$ & -1.02 & $(0.26)$ & -1.09 & $(0.32)$ & -0.91 & $(0.27)$ & $-1.18(0.29)$ \\
\hline Katholikenanteil ${ }^{2}$ & 1.42 & $(0.27)$ & 1.32 & $(0.30)$ & 1.73 & $(0.38)$ & 1.24 & $(0.33)$ & $1.42(0.37)$ \\
\hline Katholik * Katholikenanteil & 0.81 & $(0.25)$ & 0.84 & $(0.27)$ & 0.98 & $(0.27)$ & 1.00 & $(0.24)$ & $1.41(0.36)$ \\
\hline Katholik * Katholikenanteil ${ }^{2}$ & -0.65 & $(0.27)$ & -0.76 & $(0.29)$ & -0.64 & $(0.31)$ & -0.73 & $(0.29)$ & $-1.13(0.43)$ \\
\hline
\end{tabular}

Anmerkung: eigene Berechnung auf Basis des Forsa-Bus 1995-2010; unstandardisierte Koeffizienten (logits) aus logistischen Mehrebenenmodellen; Standardfehler in Klammern; Konstante und Varianzkomponenten nicht dargestellt.

Mit Blick auf die erste Spezifikation zeigt sich für alle fünf Bundestagswahlen eine statistisch signifikante Interaktion zwischen der individuellen Konfessionszugehörigkeit und der konfessionellen Zusammensetzung des Kontexts auf das Wahlverhalten. Katholiken wählen umso eher eine der beiden Unionsparteien, je höher ihr Anteil in einem Kreis ist. Dieser Befund steht im Einklang mit den in Hypothese 1 zusammengefassten Aussagen des wahlsoziologischen Gesetzes der sozialen Schwerkraft sowie der religionssoziologischen Höllenfeuer-Hypothese. Interessanterweise hat sich die Stärke dieses politischen Einflusses des katholischen Umfelds von 1994 auf 2009 sogar verdoppelt: 0.28 gegenüber 0.55. Entgegen allgemeiner Ansichten zu Säkularisierungs- und Individualisierungsprozessen sowie der spezifischen empirischen Befunde von Klein und Pötschke (2000) und Klein (2007), hat das katholische Sozialmilieu damit sogar an politischer Bedeutung gewonnen. Weiterhin ist anzumerken, dass Nicht-Katholiken - mit der Ausnahme der Bundestagswahl 2002 - in ihrem Wahlverhalten kaum in systematischer Weise vom regionalen Katholikenanteil beeinflusst werden. ${ }^{7}$

Der in der zweiten Modellspezifikation angenommene quadratische Zusammenhang zwischen individuellem Katholikeneffekt und kontextuellem Katholikenanteil wird von den empirischen Daten ebenfalls unterstützt. Für alle fünf Bundestagswahlen zeigen sich statistisch signifikante Cross-Level-Interaktionen zwischen individueller katholischer Zugehörigkeit und Katholikenanteil sowie zwischen individueller Zugehörigkeit und quadriertem Katholikenanteil. Während der erste Interaktionsterm positiv ist, weist der zweite ein negatives Vorzei-

7 Möglicherweise ist dieser Effekt auf die damalige Kandidatenkonstellation mit Edmund Stoiber als Kanzlerkandidat der Union zurückzuführen. Arzheimer und Schoen (2007, S. 102) führen an, dass die Union bei der Wahl 2002 durch die Kandidatur von Edmund Stoiber im katholisch geprägten Bayern ein besonders starkes Ergebnis erzielen konnte. Vermutlich haben hier auch NichtKatholiken stärker für ihren damaligen Ministerpräsidenten gestimmt. Der Kontexteffekt auf Nicht-Katholiken bei der Bundestagswahl 2002 könnte also ein „Bayern-Effekt“ sein. 
chen auf. Der individuelle Katholikeneffekt auf die Wahl der Unionsparteien steigt demnach zunächst mit dem Katholikenanteil im sozialen Umfeld an und schwächt sich dann aber bei weiter wachsender katholischer Dominanz wieder ab. Dieser Befund eines nicht-linearen Kontexteffekts steht in Einklang mit unserer Hypothese 2.

Auch in dieser Spezifikation erweist sich die Rolle des katholischen Kontextes für die jüngste Bundestagswahl 2009 als bedeutsamer als für 1994 (1.41 und -1.13 gegenüber 0.81 und -0.65$)$. Interessanterweise zeigt sich nun auch ein Effekt des katholischen Umfelds auf das Wahlverhalten von Nicht-Katholiken und auch dieser ist nicht-linearer Natur, allerdings in umgekehrter Richtung. Die Wahrscheinlichkeit für Nicht-Katholiken, eine der Unionsparteien zu wählen, nimmt mit steigendem Katholikenanteil zunächst ab und dann wieder zu. Dies spiegelt die These der religiösen Polarisierung unter umgekehrten Vorzeichen.

Prinzipiell werden sowohl die lineare Kontexthypothese 1 als auch die quadratische Kontexthypothese 2 durch die Daten gestützt. Um zu entscheiden, welche der beiden Modellspezifikationen nun die bessere Beschreibung der sozialen Prozesse im katholischen Milieu liefert, vergleichen wir die Modellgüte der beiden Modellvarianten (gemessen an der Devianz) und testen, inwieweit die Aufnahme eines quadratischen Interaktionsterms die Modellanpassung signifikant verbessert. Wie Tabelle 3 zeigt, weist die quadratische Spezifikation bei allen fünf Bundestagswahlen eine niedrigere Devianz und damit eine bessere Anpassung auf. Den Likelihood-Ratio-Tests zufolge ist diese Verbesserung in der Modellgüte auch in allen fünf Fällen signifikant. Mit anderen Worten ist das quadratische Kontextmodell dem linearen vorzuziehen. Kontextuelle Effekte auf die politische Wirksamkeit sozialer Identitäten verlaufen nicht linear.

Tabelle 3: Modellvergleich für zwei verschiedene Spezifikationen des Kontexteffekts

\begin{tabular}{llllll}
\hline & & & \multicolumn{2}{c}{ LR-Test } \\
\hline Bundestagswabl & Modell & Devianz & DF & $\chi^{2}(\mathrm{DF})$ & $\operatorname{Pr}\left(>\chi^{2}\right)$ \\
\hline 1994 & Linear & 401079.0 & 7 & & \\
& Quadratisch & 401051.5 & 9 & $27.442(2)$ & 0.000 \\
\multirow{2}{*}{2098} & Linear & 351437.2 & 7 & & \\
& Quadratisch & 351418.2 & 9 & \multirow{2}{*}{$19.008(2)$} & 0.000 \\
2005 & Linear & 324257.5 & 7 & & \\
& Quadratisch & 324237.7 & 9 & $19.779(2)$ & 0.000 \\
& Linear & 431842.9 & 7 & & \\
& Quadratisch & 431828.4 & 9 & $14.523(2)$ & 0.001 \\
& Linear & 141753.2 & 7 & & \\
& Quadratisch & 141738.4 & 9 & $14.777(2)$ & 0.001 \\
\hline
\end{tabular}

Anmerkung: Modelle aus Tabelle 2 
Zur besseren Verdeutlichung der quadratischen Cross-Level-Interaktion sind in Abbildung 4 die parametrischen Regressionslinien der Modellgleichung für die varying slopes zusammen mit den tatsächlichen Koeffizienten grafisch dargestellt. Für alle fünf Bundestagswahlen zeigt sich eine klare umgekehrt u-förmige Kurve, welche der Aussage von Hypothese 2 entspricht: Der individuelle Katholikeneffekt auf die Wahl der Unionsparteien steigt mit zunehmendem Katholikenanteil zunächst an und nimmt dann wieder ab. Während die tatsächlich beobachteten Werte für die Jahre 1994 und 1998 noch relativ stark um die Regressionskurve streuen, liegen sie für die Jahre 2002 bis 2009 äußerst dicht an der quadratischen Regressionsgleichung, was für eine sehr gute Beschreibung der kontextuellen Variation des individuellen Katholikeneffekts spricht.

\section{Abbildung 4: Quadratische Modellierung für den individuellen Katholikeneffekt (Punkte: tatsächliche Effekte [shrinkage estimates]; schwarze Linie: Vorhersage des Modells)}
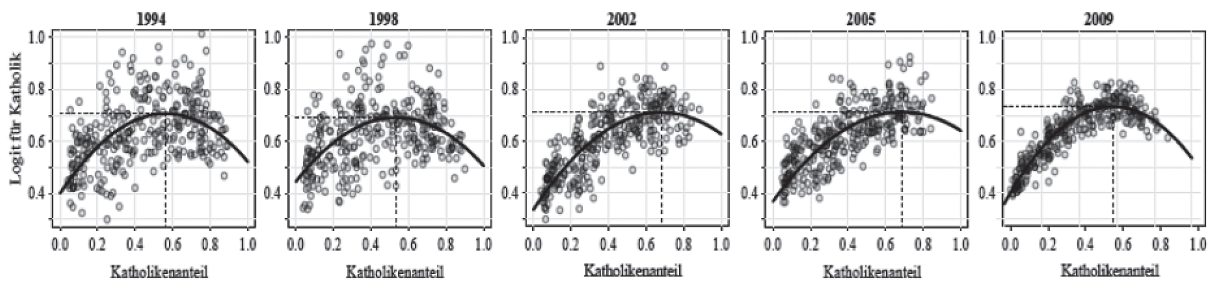

Quelle: eigene Darstellung auf Basis der in Tabelle 2 dargestellten Schätzungen (quadratische Modellierung)

Mittels der parametrischen Modellgleichung lässt sich nun auch der tipping point berechnen und damit der kritische Schwellenwert in der Gruppengröße der Katholiken quantifizieren, ab dem die politische Wirksamkeit der individuellen Gruppenzugehörigkeit wieder abnimmt. Dieser Wert ist nichts anderes als das Maximum der Kurve, welches sich mit einfachen Werkzeugen der Analysis leicht finden lässt. Der konkrete tipping point variiert zwar über die fünf Bundestagswahlen (1994: Katholikenanteil von 63 Prozent, 1998: 55 Prozent, 2002: 77 Prozent, 2005: 67 Prozent und 2009: 62 Prozent), doch lässt er sich grob bei einer katholischen Gruppengröße von zwei Dritteln der regionalen Bevölkerung verorten. ${ }^{8}$ Sobald diese kritische Masse erreicht ist, verliert die eigene katholische Identität an politischer Wirksamkeit.

Abschließend gibt Abbildung 5 noch Aufschluss über die substanzielle Größe des variierenden Katholikeneffekts. Dargestellt sind die Differenzen zwischen Katholiken und Nicht-Katholiken in den vorhergesagten Wahrscheinlichkeiten der

8 Der besonders ausgeprägte Effekt des katholischen Milieus für die Bundestagswahl 2002 lässt sich möglicherweise auf die Kandidatur von Edmund Stoiber als Kanzlerkandidat der Unionsparteien zurückführen. Roßteutscher (2012) zufolge habe diese Nominierung für einen „Super-Katholizismus“ gestanden und bei den Protestanten „das überkommene konfessionelle Ressentiment der Protestanten “ gegen die Unionsparteien wiederbelebt. Daher sei die konfessionelle Konfliktlinie hier stärker hervorgetreten. 
Wahl der Unionsparteien in Abhängigkeit des Katholikenanteils. Die simulierten grauen Linien geben die Schätzunsicherheit wieder und die kleinen senkrechten Striche oberhalb der x-Achse zeigen die empirische Verteilung des Katholikenanteils in den 326 Kreisen an. Obgleich die Differenz in den vorhergesagten Wahrscheinlichkeiten von Bundestagswahl zu Bundestagswahl leicht variiert, lässt sich dennoch sagen, dass Katholiken in einer deutlichen Minderheitenposition von einem Bevölkerungsanteil von 5 Prozent eine um etwa 9 bis 11 Prozent höhere Wahrscheinlichkeit aufweisen, eine der beiden Unionsparteien zu wählen als Nicht-Katholiken. In einer komfortablen Mehrheit von 60 Prozent Bevölkerungsanteil liegt diese Differenz in der vorhergesagten Wahrscheinlichkeit zwischen 15 und 17 Prozent, ehe sie dann wieder abnimmt. Angesichts der teilweise sehr knappen Ergebnisse bei den vergangenen Bundestagswahlen können jedoch schon scheinbar geringe Prozentsätze für den Wahlausgang relevant sein.

Abbildung 5: Differenz in den vorhergesagten Wahrscheinlichkeiten für Katholiken und Nicht-Katholiken (schwarze Linie: Vorhersage für die Differenz; graue Linien: Simulation für Schätzunsicherheit der Vorhersage; vertikale Linien oberhalb der x-Achse: empirische Verteilung)

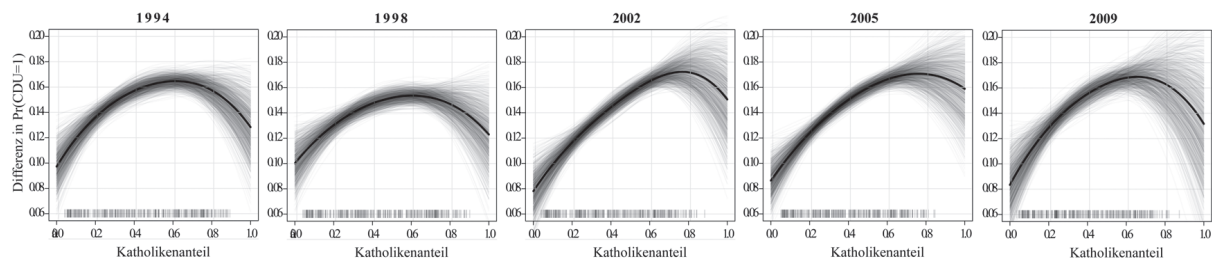

Quelle: eigene Darstellung auf Basis der in Tabelle 2 dargestellten Schätzungen (quadratische Modellierung)

\subsection{Robustheitsanalyse}

Um die Robustheit unserer Befunde zum quadratischen Kontexteffekt zu überprüfen, werden im Folgenden verschiedene alternative Modellspezifikationen getestet. Erstens kontrollieren wir für weitere Variablen sowohl auf Individual- als auch auf Kontextebene. Zweitens überprüfen wir, ob der Effekt des katholischen Kontexts auf die Wahl der Unionsparteien allein auf das katholische Bayern zurückzuführen ist. Drittens testen wir eine weitere funktionale, namentlich eine logarithmische Form für die Cross-Level-Interaktion.

In der vorangegangen Analyse haben wir bewusst eine sehr sparsame Modellspezifikation gewählt, die ausschließlich die theoretisch interessierenden Variablen beinhaltet. In ihrer deskriptiven Aussage zum kontextabhängigen Wahlverhalten von Katholiken sind diese einfachen Modelle auch vollkommen ausreichend. Dennoch sollen im Folgenden zusätzliche Größen in die Modellgleichung aufgenommen werden, die möglicherweise den Zusammenhang zwischen katholischer Konfessionszugehörigkeit und Wahlpräferenz für die Unionsparteien konfundieren. Konkret wird auf Individualebene für Geschlecht, Alter, Bildung, Kirchgang, Arbeiterstatus und Gewerkschaftsmitgliedschaft kontrolliert. Von weitaus größe- 
rer Bedeutung ist jedoch die Kontrolle weiterer Kontextgrößen, welche mit hohen Katholikenanteilen und konservativer Wahlneigung zusammenhängen. Hier ist besonders an die regionale Wirtschaftskraft sowie an den Urbanisierungsgrad zu denken. Durch die Berücksichtigung der Wirtschaftsstärke kann sichergestellt werden, dass der Effekt nicht ausschließlich durch Süddeutschland hervorgerufen wird. Hinter dem Einbezug des Urbanisierungsgrads steht die Idee, für die Besonderheiten des ländlichen Raums hinsichtlich religiöser sowie parteipolitischer Prägung zu kontrollieren. Diese beiden Faktoren werden sowohl in die Gleichung für die varying intercepts als auch in die Gleichung für die varying slopes eingeführt.

Tabelle 4 stellt die Ergebnisse unter Einbezug der genannten Kontrollvariablen dar. Wie erwartet bleiben die Resultate auch bei dieser erweiterten Spezifikation robust. Die Koeffizienten für die individuelle Konfessionszugehörigkeit schwächen sich im Vergleich zu den einfachen Modellen nur sehr geringfügig ab, am stärksten noch für das Jahr 1998 (0.39 gegenüber 0.46). Wichtiger für die Argumentation der vorliegenden Studie ist jedoch, dass der quadratische Interaktionsterm zwischen katholischer Identität und kontextuellem Katholikenanteil bestehen bleibt, wenn man die regionale Wirtschaftslage und die Ländlichkeit des Kontexts berücksichtigt. Auch die Stärke der ursprünglichen Effekte ändert sich kaum, wobei die Koeffizienten für die Jahre 1994, 1998 und 2005 sogar eher grösser werden. Lediglich die nicht-linearen Kontexteffekte für Nicht-Katholiken schwächen sich etwas ab, wobei die Schätzunsicherheit der Koeffizienten für den quadrierten Katholikenanteil 2005 und 2009 die herkömmliche Schwelle statistischer Signifikanz geringfügig überschreitet. Ansonsten lässt sich noch feststellen, dass in wirtschaftsstarken und ländlichen Kreisen eher die Unionsparteien gewählt werden. Der individuelle Katholikeneffekt ist in wirtschaftsstarken Kontexten etwas geringer (zumindest bei den Bundestagswahlen 1994, 1998 und 2005) und für 1998 und 2009 in städtischen Gebieten etwas höher. 
Tabelle 4: Kontexteffekte auf die Wahlentscheidung bei Katholiken - Robustheitsanalysen

\begin{tabular}{|c|c|c|c|c|c|}
\hline & 1994 & 1998 & 2002 & 2005 & 2009 \\
\hline \multicolumn{6}{|l|}{ Mit Kontrollvariablen } \\
\hline Individuelle Kontrollvariablen ${ }^{\mathrm{a}}$ & $j a$ & $j a$ & $j a$ & ja & $j a$ \\
\hline Katholik & $0.42(0.06)$ & $0.39(0.06)$ & $0.32(0.06)$ & $0.33(0.05)$ & $0.29(0.08)$ \\
\hline Katholikenanteil & $-0.93(0.20)$ & $-0.76(0.22)$ & $-0.62(0.27)$ & $-0.49(0.22)$ & $-0.68(0.25)$ \\
\hline Katholikenanteil ${ }^{2}$ & $0.88(0.24)$ & $0.83(0.26)$ & $0.90(0.33)$ & $0.55(0.28)$ & $0.64(0.33)$ \\
\hline Katholik * Katholikenanteil & $1.04(0.25)$ & $0.96(0.26)$ & $0.92(0.27)$ & $1.11(0.24)$ & $1.23(0.35)$ \\
\hline Katholik * Katholikenanteil ${ }^{2}$ & $-0.92(0.28)$ & $-0.83(0.29)$ & $-0.58(0.32)$ & $-0.85(0.29)$ & $-0.94(0.43)$ \\
\hline BIP pro Kopf & $0.08(0.02)$ & $0.08(0.01)$ & $0.08(0.02)$ & $0.03(0.01)$ & $0.04(0.01)$ \\
\hline Urbanisierungsgrad & $-0.09(0.01)$ & $-0.10(0.01)$ & $-0.12(0.01)$ & $-0.09(0.01)$ & $-0.08(0.01)$ \\
\hline Katholik * BIP pro Kopf & $-0.04(0.02)$ & $-0.04(0.02)$ & $-0.01(0.01)$ & $-0.02(0.01)$ & $-0.02(0.02)$ \\
\hline Katholik * Urbanisierungsgrad & $0.01(0.01)$ & $0.03(0.01)$ & $0.01(0.01)$ & $0.01(0.01)$ & $0.03(0.01)$ \\
\hline \multicolumn{6}{|l|}{ Obne Bayern } \\
\hline Katholik & $0.50(0.06)$ & $0.45(0.06)$ & $0.39(0.06)$ & $0.40(0.05)$ & $0.35(0.08)$ \\
\hline Katholikenanteil & $-1.34(0.27)$ & $-1.31(0.29)$ & $-1.19(0.32)$ & $-1.03(0.30)$ & $-1.50(0.32)$ \\
\hline Katholikenanteil ${ }^{2}$ & $1.32(0.34)$ & $1.44(0.37)$ & $1.28(0.43)$ & $1.10(0.41)$ & $1.74(0.44)$ \\
\hline Katholik * Katholikenanteil & $0.52(0.30)$ & $1.05(0.32)$ & $0.81(0.31)$ & $0.82(0.29)$ & $1.32(0.42)$ \\
\hline Katholik * Katholikenanteil ${ }^{2}$ & $-0.19(0.35)$ & $-1.01(0.38)$ & $-0.41(0.39)$ & $-0.39(0.36)$ & $-1.05(0.54)$ \\
\hline \multicolumn{6}{|l|}{ Logarithmisch } \\
\hline Katholik & $0.75(0.02)$ & $0.73(0.02)$ & $0.79(0.02)$ & $0.78(0.02)$ & $0.85(0.03)$ \\
\hline $\log ($ Katholikenanteil $)$ & $-0.03(0.02)$ & $0.00(0.02)$ & $0.06(0.02)$ & $0.00(0.02)$ & $-0.05(0.02)$ \\
\hline $\begin{array}{l}\text { Katholik" } \\
\log (\text { Katholikenanteil })\end{array}$ & $0.10(0.02)$ & $0.09(0.02)$ & $0.16(0.02)$ & $0.14(0.02)$ & $0.18(0.03)$ \\
\hline
\end{tabular}

Anmerkung: eigene Berechnung auf Basis des Forsa-Bus 1995-2010; unstandardisierte Koeffizienten (logits) aus logistischen Mehrebenenmodellen; Standardfehler in Klammern; Konstante, individuelle Kontrollvariablen und Varianzkomponenten nicht dargestellt; a Geschlecht, Alter, Bildung, Kirchgang, Arbeiterstatus und Gewerkschaftsmitgliedschaft.

Auch für die Modelle mit individuellen und kontextuellen Kontrollvariablen lässt sich die relative Modellgüte zwischen einer linearen und quadratischen Modellierung der Kontexteffekte vergleichen. Wie die Devianzen und Likelihood-RatioTests in Tabelle 7 im Anhang zeigen, stellt die nicht-lineare Modellierung auch unter Kontrolle weiterer Faktoren eine signifikante Verbesserung gegenüber der linearen dar und ist dieser vorzuziehen. Lediglich mit den Daten für die Bundestagswahl 2009 liegt der entsprechende p-Wert knapp über den konventionellen 0.05 , was jedoch kaum von Belang ist.

Um in einem zweiten Schritt die Möglichkeit auszuschließen, dass der gefundene Zusammenhang von katholischem Sozialmilieu und Wahlpräferenz für die Unionsparteien lediglich auf Bayern und dessen kulturelle Eigenheiten zurückzuführen ist, entfernen wir in einem weiteren Robustheitstest alle bayerischen Landkreise und kreisfreien Städte aus unserem Sample. Für dieses reduzierte Sample 
mit J = 230 Kreisen rechnen wir wieder mit der ursprünglichen sparsamen Modellspezifikation. Wie die Ergebnisse in Tabelle 4 zeigen, lassen sich unsere Befunde zur Rolle katholischer Kontexte nicht allein auf Bayern zurückführen, sondern bleiben auch unter Ausschluss der bayrischen Kreise weitgehend robust. Der individuelle Katholikeneffekt wird mit der Ausnahme von 1998 sogar leicht stärker. Der quadratische Interaktionsterm schwächt sich dagegen wiederum mit Ausnahme von 1998 etwas ab. Bei den Bundestagswahlen ab 2002 sind die Koeffizienten für den Interaktionsterm mit dem quadrierten Katholikenanteil zusätzlich mit höherer Schätzunsicherheit behaftet und verlieren ihre statistische Signifikanz. Das generelle Muster bleibt allerdings bestehen. Schließlich erweisen sich auch die quadratischen Kontexteffekte für Nicht-Katholiken als robust.

In Tabelle 8 im Anhang ist wiederum der Modellvergleich zwischen linearer und quadratischer Spezifikation der Cross-Level-Interaktion aufgeführt. Auch unter Ausschluss von Bayern weist die quadratische Modellierung eine signifikant bessere Anpassung an die empirischen Daten auf, als dies für die lineare der Fall ist. Das gilt insbesondere auch für die Jahre 2002, 2005 und 2009.

Als dritten Robustheitstest kontrastieren wir unsere quadratische Modellierung mit einer alternativen funktionalen Form der Cross-Level-Interaktion, indem wir den Katholikenanteil logarithmieren. Auf diese Weise lässt sich ebenfalls eine nicht-lineare Beziehung zwischen individueller katholischer Identität und katholischem Kontext abbilden, welche jedoch einer anderen theoretischen Aussage zum sozialen Einflussprozess entsprechen würde. Ab einer gewissen Gruppengröße würde die politische Wirksamkeit der sozialen Identität zwar ebenfalls nicht weiter zunehmen. Aber anstatt wieder abzunehmen, wie das quadratische Modell unterstellt, würde sich die Wirkung auf einem bestimmten Niveau stabilisieren.

Die Ergebnisse in Tabelle 4 machen deutlich, dass diese theoretische Alternative durchaus von den empirischen Daten gestützt wird. Für alle fünf Bundestagswahlen ergibt sich ein statistisch signifikanter Interaktionsterm zwischen individueller Konfessionszugehörigkeit und logarithmiertem Katholikenanteil. Allerdings zeigt der Modellvergleich in Tabelle 9 im Anhang, dass die quadratische Spezifikation stets signifikant besser auf die Daten passt. Auch dieser Befund spricht für unser ursprüngliches Modell.

\section{Schluss}

Während die prägende Kraft von Religion für die deutsche Parteienlandschaft und das politische Verhalten der Bürger lange Zeit unumstritten war, konzentriert sich das wissenschaftliche Interesse aktuell auf die Frage nach der Stabilität und dem Wandel der konfessionellen Konfliktlinie im Wahlverhalten (Arzheimer u. Schoen 2007; Elff u. Roßteutscher 2011; Roßteutscher 2012; Schoen 2009). Wenngleich ein positiver Effekt der katholischen Konfession auf die Wahrscheinlichkeit einer Wahlentscheidung für die Unionsparteien auf Individualebene nach wie vor als gesichert gelten kann, sind die dafür verantwortlichen sozialen Prozesse kaum erforscht und die rar gesäten Befunde wenig eindeutig. Die vorliegende Studie leistet sowohl einen theoretischen als auch empirischen Beitrag zu dieser 
Debatte und zeigt, dass der Zusammenhang zwischen Religionszugehörigkeit und politischem Verhalten entscheidend durch den sozialen Kontext moderiert wird.

Im Gegensatz zu herkömmlichen wahl- und religionssoziologischen Theorien, die einen linearen Kontexteffekt annehmen, sagt unser theoretisches Argument einen nicht-linearen Kontexteffekt vorher. Entgegen dem Gesetz der sozialen Schwerkraft und der Höllenfeuer-Hypothese nimmt der Einfluss von Religion mit steigender Gruppengröße nicht stetig zu, sondern sinkt wieder, sobald die eigene Religionsgruppe die überwiegende Mehrheit im sozialen Kontext stellt. In einer solchen Situation dient die religiöse Gegengruppe nicht mehr als Gegenstand der sozialen Abgrenzung und des politischen Wettbewerbs. Religion als sozialstrukturelles Merkmal verliert dann seine politisch wirksame Kraft.

Die empirischen Ergebnisse geben unserem theoretischen Argument recht und weisen auf einen nicht-linearen Moderatoreffekt des sozialen Kontextes auf den Individualzusammenhang zwischen Konfession und Wahlentscheidung hin. Religion ist also vor allem dann politisch salient, wenn wir einen religiös polarisierten Kontext vorfinden. Bis zu einem Katholikenanteil von circa 60 Prozent im Kontext wird die Wirksamkeit des Individualmerkmals verstärkt. Dann ist der tipping point erreicht und die Stärke des Individualeffekts nimmt bei weiterer Zunahme des Katholikenanteils ab. In einem stark katholisch geprägten Kontext entfaltet die Konfession schlicht keine identitätsstiftende Wirkung mehr. Dies entspricht auch dem Befund von Geser (1997), wonach gerade in stark katholisch geprägten Kontexten der Anreiz, eine spezifische katholische Kultur zu entwickeln, fehlt.

Die nicht-lineare Spezifikation des Kontexteffekts passt für alle fünf betrachteten Bundestagswahlen von 1994 bis 2009 besser an die Daten als die lineare. Auch zahlreiche Robustheitstests, namentlich die Berücksichtigung von Kontrollvariablen auf Individual- und Kontextebene, die Schätzung ohne Bayern sowie eine alternative Modellierung der Effekte, bestätigen dieses Ergebnis. Diese Befunde sprechen - entgegen der Feststellung von Klein und Pötschke (2000) - dafür, dass keineswegs von einer abnehmenden Prägekraft des katholischen Milieus ausgegangen werden kann, sondern die traditionelle katholische Wahlnorm hier nach wie vor wirksam aufrechterhalten wird. Interessanterweise nimmt die Stärke des moderierenden Kontexteffektes über Zeit sogar zu und erreicht für die letzte Wahl 2009 den höchsten Wert.

Wenngleich unsere Argumentation vor allem auf Gruppenprozesse der Identitätsentwicklung und Normverstärkung verweist und somit soziologisch inspiriert ist, zeigt die zentrale Rolle, welche wir dem konfessionellen Wettbewerb und den dadurch erzeugten Anreizen zuschreiben, auch die prinzipielle Anschlussfähigkeit an stärker ökonomisch ausgerichtete Erklärungsmodelle des Wahlverhaltens (Arzheimer u. Schmitt 2005; Blais 2000; Downs 1957). Aufgrund der konfessionsbedingten Parteibindung wäre in einem konfessionell heterogenen Kontext auch ein stärkerer Wettbewerb zwischen den Parteien zu erwarten. Da die eigene Stimme für die präferierte Partei bei ausgeprägtem Wettbewerb als potenziell entscheidend wahrgenommen werden kann, lässt sich der Individualeffekt von Religion durchaus auch auf das strategisch-rationale Verhalten der beteiligten Akteure zurückführen. Unsere Studie ist damit ein Beispiel für die mögliche und wünschenswerte Konvergenz verschiedener Erklärungsansätze der Wahlforschung hin zu ei- 
nem umfassenderen „model of citizens who act purposefully on the basis of their own goals, ambitions and needs" (Carmines u. Huckfeldt 1998, S. 248; siehe dazu auch Abrams et al. 2011).

Mit Blick auf die in der Religionssoziologie seit geraumer Zeit geführte Säkularisierungsdebatte scheinen unsere Ergebnisse den Erwartungen der klassischen Säkularisierungstheorie zu widersprechen. Weder beobachten wir einen Verlust der sozialen oder öffentlichen Bedeutung von Religion, noch führt religiöse Konkurrenz im sozial-räumlichen Kontext zu einer Abschwächung religiöser Identitäten (Berger 1990; Bruce 2002; Pickel 2010). Sowohl theoretisch als auch empirisch ist genau das Gegenteil der Fall. In Einklang mit den theoretischen Argumenten der religionsökonomischen Schule steht der konfessionelle Wettbewerb vielmehr mit erhöhter religiöser Mobilisierung und damit einer verstärkten sozialen Wirksamkeit von Religion in Verbindung (Iannaccone 1991; Stark u. Finke 2000). Dies ist insofern bemerkenswert, als die empirische Evidenz für das religiöse Marktmodell bislang uneinheitlich und insbesondere im europäischen Kontext eher dürftig ausfällt (Chaves u. Gorski 2001). Da religiöse Polarisierung und damit der konfessionelle Wettbewerbscharakter eines Kontexts in einem quadratischen Zusammenhang mit dem in der einschlägigen Literatur dominierenden Fraktionalisierungsmaß steht, könnte eine stärkere Berücksichtigung nicht-linearer Modellspezifikationen auch dieser Forschungstradition neue theoretische und empirische Impulse geben.

Jenseits der Bedeutung für gegenwärtige wahl- und religionssoziologische Diskussionen sind unsere Befunde für das generelle Verständnis der sozialen Strukturiertheit politischen Verhaltens relevant. Ob jemand zur Wahl geht, welche Partei er wählt oder ob er auf andere Art am politischen Leben teilhat, hängt zwar von sozialstrukturellen Merkmalen wie der Konfession, dem Bildungsstand, dem Alter oder dem Beruf einer Person ab. Wie stark und auf welche Art und Weise diese Merkmale auf das politische Verhalten einer Person wirken, ist aber selbst sozial strukturiert und wird entscheidend durch die Zusammensetzung des sozialen Umfelds beeinflusst. Diese sozialen Einflussprozesse müssen nicht zwangsläufig linear verlaufen, sondern können - wie wir zeigen - durchaus einer komplexeren und interessanteren Logik folgen.

\section{Literatur}

Abrams, Samuel, Torben Iversen, und David Soskice. 2011. Informal Social Networks and Rational Voting. British Journal of Political Science 41: 229-257. doi: 10.1017/ S0007123410000499.

Arzheimer, Kai. 2009. Contextual Factors and the Extreme Right Vote in Western Europe, 1980-2002. American Journal of Political Science 53: 259-275. doi: 10.1111/j.15405907.2009.00369.x.

Arzheimer, Kai, und Annette Schmitt. 2005. Der ökonomische Ansatz. In Handbuch Wablforschung, Hrsg. Jürgen W. Falter und Harald Schoen, 243-304. Wiesbaden: VS Verlag für Sozialwissenschaften.

Arzheimer, Kai, und Harald Schoen. 2007. Mehr als eine Erinnerung an das 19. Jahrhundert? Das sozio-ökonomische und das religiös-konfessionelle Cleavage und Wahlverh- 
alten 1994-2005. In Der gesamtdeutsche Wähler. Stabilität und Wandel des Wählerverhaltens im wiedervereinigten Deutschland, Hrsg. Hans Rattinger, Oscar W. Gabriel und Jürgen W. Falter, 89-112. Baden-Baden: Nomos.

Baldassarri, Delia. 2009. Social Networks, Political Heterogeneity, and Interpersonal Influence. Evidence from the 2006 Italian Elections. http://vanity.dss.ucdavis.edu/ maoz/ Networks\%20Workshop/baldassarri2009a.pdf Zugegriffen: 27.12.2012.

Bearman, Peter S., und Hannah Brückner. 2001. Promising the Future: Virginity Pledges and First Intercourse. American Journal of Sociology 106: 859-912. doi: 10.1086/ 320295.

Berelson, Bernard R., Paul F. Lazarsfeld, und William N. McPhee. 1954. Voting. A Study of Opinion Formation in a Presidential Campaign. Chicago: University of Chicago Press. Berger, Peter L. 1990. The Sacred Canopy. Elements of a Sociological Theory of Religion. New York: Doubleday.

Blais, André. 2000. To Vote or Not to Vote? The Merits and Limits of Rational Choice Theory. Pittsburgh: University of Pittsburgh Press.

Blau, Peter M. 1977. A Macrosociological Theory of Social Structure. The American Journal of Sociology 83: 26-54. doi: 10.1086/226505.

Blau, Peter M., und Joseph E. Schwartz. 1984. Crosscutting Social Circles. Testing a Macrostructural Theory of Intergroup Relations. Orlando: Academic Press.

Books, John W., und Charles L. Prysby. 1991. Political Behavior and the Local Context. New York: Praeger.

Brettschneider, Frank, Jan van Deth, und Edeltraud Roller (Hrsg.). 2002. Das Ende der politisierten Sozialstruktur? Opladen: Leske + Budrich.

Brewer, Marilynn B. 1999. The Psychology of Prejudice: Ingroup Love or Outgroup Hate? Journal of Social Issues 55: 429-444. doi: 10.1111/0022-4537.00126.

Brooks, Clem, Paul Nieuwbeerta, und Jeff Manza. 2004. Cleavage-based voting behavior in cross-national perspective: evidence from six postwar democracies. Social Science Research 35: 88-128. doi: 10.1016/j.ssresearch.2004.06.005.

Bruce, Steve. 2002. God is Dead. Secularization in the West. Oxford: Malden.

Bühlmann, Marc. 2006. Politische Partizipation im kommunalen Kontext. Der Einfluss lokaler Kontexteigenschaften auf individuelles politisches Partizipationsverhalten. Bern: Haupt.

Bühlmann, Marc, und Markus Freitag. 2006. Individual and contextual determinants of electoral participation. Swiss Political Science Review 12: 13-47. doi: 10.1002/j.16626370.2006.tb00059.x.

Burbank, Matthew J. 1997. Explaining Contextual Effects of Vote Choice. Political Behavior 19: 113-132. doi: 10.1023/A:1024806024732.

Campbell, David E. (Hrsg.). 2007. A Matter of Faith. Religion in the 2004 Presidential Election. Washington, D. C.: Brookings Institution Press.

Carmines, Edward G., und Robert R. Huckfeldt. 1998. Political Behavior: An Overview. In A New Handbook of Political Science, Hrsg. Robert Goodin und Hans-Dieter Klingemann, 223-254. Oxford: Oxford University Press.

Chaves, Mark, und Philip Gorski. 2001. Religious Pluralism and Religious Participation. Annual Review of Sociology 27: 261-281. doi: 10.1146/annurev.soc.27.1.261. 
Chung, Yeojin, Sophia Rabe-Hesketh, Vincent Dorie, Andrew Gelman, und Jingchen Liu. 2013. A Nondegenerate Penalized Likelihood Estimator for Variance Parameters in Multilevel Models. Psychometrika 78: 685-709.

Cleveland, William S. 1979. Robust Locally Weighted Regression and Smoothing Scatterplots. Journal of the American Statistical Association 74: 829-836. doi: 10.2307/ 2286407.

Debus, Marc. 2010. Soziale Konfliktlinien und Wahlverhalten: Eine Analyse der Determinanten der Wahlabsicht bei Bundestagswahlen von 1969 bis 2009. Kölner Zeitschrift für Soziologie und Sozialpsychologie 62: 731-749. doi: 10.1007/s11577-010-0114-1.

Dorie, Vincent. 2011. Bayesian Linear Mixed-Effects models. R package version 0.01-4.

Downs, Anthony. 1957. An Economic Theory of Democracy. New York: Harper \& Brothers.

Dülmer, Hermann. 2005. Die Schätzung von kleinräumigen Kontextinformationen aus Umfragedaten. In Deutschland regional. Sozialwissenschaftliche Daten im Forschungsverbund, Hrsg. Gerd Grözinger und Wenzel Matiaske, 29-39. München: Rainer Hampp.

Dülmer, Hermann, und Dieter Ohr. 2008. Rechtsextremistische Wahlabsicht und regionaler Kontext: Mehrebenenanalysen zur Rolle sozialer Milieus und regionaler Gruppenkonflikte in Deutschland. Politische Vierteljahresschrift 49: 491-517. doi: 10.1007/ s11615-008-0108-z.

Dyck, Joshua J., Brian J. Gaines, und Daron R. Shaw. 2009. The Effect of Local Political Context on How Americans Vote. American Politics Research 37: 1088-1115. doi: 10.1177/1532673X09332932.

Elff, Martin. 2007. Social Structure and Electoral Behavior in Comparative Perspective: The Decline of Social Cleavages in Western Europe Revisited. Perspectives on Politics 5: 277-294. doi: 10.1017/S1537592707070788.

Elff, Martin, und Sigrid Roßteutscher. 2011. Stability or Decline? Class, Religion and the Vote in Germany. German Politics 20: 107-127. doi: 10.1080/09644008.2011.554109.

Essmer, Yilmaz, und Thorleif Pettersson. 2007. The Effects of Religion and Religiosity on Voting Behavior. In The Oxford Handbook of Political Behavior, Hrsg. Russell J. Dalton und Hans-Dieter Klingemann, 481-503. Oxford: Oxford University Press.

Fiorina, Morris P. 2005. Culture War? The Myth of a Polarized America. New York: Longman.

Forsa. 1998. Forsa-Bus 1995. ZA-Nr. 2983. Datenfile Version 1.0.0. Berlin: forsa Gesellschaft für Sozialforschung und statistische Analyse mbH. doi: 10.4232/1.2983.

Forsa. 1998. Forsa-Bus 1996. ZA-Nr. 2984. Datenfile Version 1.0.0. Berlin: forsa Gesellschaft für Sozialforschung und statistische Analyse mbH. doi: 10.4232/1.2984.

Forsa. 1998. Forsa-Bus 1997. ZA-Nr. 2985. Datenfile Version 1.0.0. Berlin: forsa Gesellschaft für Sozialforschung und statistische Analyse mbH. doi: 10.4232/1.2985.

Forsa. 1999. Forsa-Bus 1998. ZA-Nr. 3162. Datenfile Version 1.0.0. Berlin: forsa Gesellschaft für Sozialforschung und statistische Analyse mbH. doi: 10.4232/1.3162.

Forsa. 2000. Forsa-Bus 1999. ZA-Nr. 3289. Datenfile Version 1.0.0. Berlin: forsa Gesellschaft für Sozialforschung und statistische Analyse mbH. doi: 10.4232/1.3289.

Forsa. 2001. Forsa-Bus 2000. ZA-Nr. 3486. Datenfile Version 1.0.0. Berlin: forsa Gesellschaft für Sozialforschung und statistische Analyse mbH. doi: 10.4232/1.3486.

Forsa. 2002. Forsa-Bus 2001. ZA-Nr. 3675. Datenfile Version 1.0.0. Berlin: forsa Gesellschaft für Sozialforschung und statistische Analyse mbH. doi: 10.4232/1.3675. 
Forsa. 2012. Forsa-Bus 2002. ZA-Nr. 3909. Datenfile Version 2.0.0. Berlin: forsa Gesellschaft für Sozialforschung und statistische Analyse mbH. doi: 10.4232/1.11146.

Forsa. 2012. Forsa-Bus 2003. ZA-Nr. 4070. Datenfile Version 2.0.0. Berlin: forsa Gesellschaft für Sozialforschung und statistische Analyse mbH. doi: 10.4232/1.11147.

Forsa. 2012. Forsa-Bus 2004. ZA-Nr. 4192. Datenfile Version 2.0.0. Berlin: forsa Gesellschaft für Sozialforschung und statistische Analyse mbH. doi: 10.4232/1.11148.

Forsa. 2012. Forsa-Bus 2005. ZA-Nr. 4343. Datenfile Version 2.0.0. Berlin: forsa Gesellschaft für Sozialforschung und statistische Analyse mbH. doi: 10.4232/1.11150.

Forsa. 2007. Forsa-Bus 2006. ZA-Nr. 4514. Datenfile Version 1.0.0. Berlin: forsa Gesellschaft für Sozialforschung und statistische Analyse mbH. doi: 10.4232/1.4514.

Forsa. 2008. Forsa-Bus 2007. ZA-Nr. 4552. Datenfile Version 1.0.0. Berlin: forsa Gesellschaft für Sozialforschung und statistische Analyse mbH. doi: 10.4232/1.4552.

Forsa. 2009. Forsa-Bus 2008. ZA-Nr. 4876. Datenfile Version 1.0.0. Berlin: forsa Gesellschaft für Sozialforschung und statistische Analyse mbH. doi: 10.4232/1.4876.

Forsa. 2010. Forsa-Bus 2009. ZA-Nr. 5049. Datenfile Version 1.0.0. Berlin: forsa Gesellschaft für Sozialforschung und statistische Analyse mbH. doi: 10.4232/1.10117.

Forsa. 2011. Forsa-Bus 2010. ZA-Nr. 5293. Datenfile Version 1.0.0. Berlin: forsa Gesellschaft für Sozialforschung und statistische Analyse mbH. doi: 10.4232/1.10703.

Geser, Hans. 1997. The Persistence of Confessional Political Cultures in Switzerland: Some Puzzling Empirical Evidence from Local Parties. Swiss Political Science Review 3: 1-29. doi: 10.1002/j.1662-6370.1997.tb00202.x.

Gelman, Andrew, und Gary King. 1993. Why Are American Presidential Election Polls so Variable when Votes Are so Predictable? British Journal of Political Science 23: 409451. doi: 10.1017/S0007123400006682.

Gelman, Andrew, und Jennifer Hill. 2007. Data Analysis Using Regression and Multilevel/ Hierarchical Models. Cambridge: Cambridge University Press.

Gelman, Andrew, David Park, Boris Shor, Joseph Bafumi, und Jeronimo Cortina. 2008. Red State, Blue State, Rich State, Poor State: Why Americans Vote the Way They Do. Princeton: Princeton University Press.

Gimpel, James G., Joshua J. Dyck, und Daron R. Shaw. 2004. Registrants, Voters, and Turnout Variability Across Neighborhoods. Political Behavior 26: 343-375. doi: 10.1007/s11109-004-0900-4.

Gimpel, James G., und J. Celeste Lay. 2005. Party Identification, Local Partisan Contexts, and the Acquisition of Participatory Attitudes. In The Social Logic of Politics. Personal Networks as Contexts for Political Behavior, Hrsg. Alan S. Zuckerman, 209-288. Philadelphia: Temple University Press.

Green, John C. 2010. The Faith Factor. How Religion Influences American Elections. Washington, D. C.: Potomac Books.

Huckfeldt, Robert R. 1979. Political Participation and the Neighborhood Social Context. American Journal of Political Science 23: 579-592. doi: 10.2307/2111030.

Huckfeldt, Robert R. 1980. Variable responses to neighborhood social contexts: Assimilation, conflict, and tipping points. Political Behavior 2: 231-257. doi: 10.1007/ BF00990481.

Huckfeldt, Robert R. 1983. Social Contexts, Social Networks, and Urban Neighborhoods: Environmental Constraints on Friendship Choice. American Journal of Sociology 89: 651-669. doi: 10.1086/227908. 
Huckfeldt, Robert R. 2007. Information, Persuasion, and Political Communication Networks. In Oxford Handbook of Political Behavior, Hrsg. Russell J. Dalton und HansDieter Klingemann, 100-122. Oxford/New York: Oxford University Press.

Huckfeldt, Robert R. 2009. Citizenship in Democratic Politics: Density Dependence and the Micro-Macro Divide. In Comparative Politics. Rationality, Culture, and Structure, Hrsg. Marc Irving Lichbach und Alan S. Zuckerman, 291-313. Cambridge: Cambridge University Press.

Huckfeldt, Robert R., und John Sprague. 1987. Networks in Context: The Social Flow of Political Information. American Political Science Review 81: 1197-1216. doi: 10.2307/ 1962585.

Huckfeldt, Robert R., und John Sprague. 1993. Citizens contexts and politics. In Political Science: The State of the Discipline II, Hrsg. Ada W. Finifter, 281-303. Washington D. C.: American Political Science Association.

Hunter, James D. 1991. Culture Wars. The Struggle to Define America. New York: Basic Books.

Iannaccone, Laurence. 1991. The Consequences of Religious Market Structure. Adam Smith and the Economics of Religion. Rationality and Society 3: 156-177. doi: 10.1177/1043463191003002002.

Jagodzinski, Wolfgang, und Markus Quandt. 2000. Religion und Wahlverhalten in der längsschnittlichen Entwicklung. In 50 Jahre Empirische Wablforschung in Deutschland. Entwicklung, Befunde, Perspektiven, Daten, Hrsg. Markus Klein, Wolfgang Jagodzinski, Ekkehard Mochmann und Dieter Ohr, 159-181. Wiesbaden: Westdeutscher Verlag.

Johnston, Ron, und Charles Pattie. 2006. Putting Voters in their Place. Geography and Elections in Great Britain. Oxford: Oxford University Press.

Jung, Matthias, Yvonne Schroth, und Andrea Wolf. 2010. Wählerverhalten und Wahlergebnis. Regierungswechsel ohne Wechselstimmung. In Die Bundestagswabl 2009. Analysen der Wahl-, Parteien-, Kommunikations- und Regierungsforschung, Hrsg. KarlRudolf Korte, 35-47. Wiesbaden: VS Verlag für Sozialwissenschaften.

Klein, Markus. 2007. Individuelles Wählerverhalten und sozialer Kontext: Logistische Mehrebenenanalysen der Wahlentscheidungen bei den Bundestagswahlen 1994, 1998 und 2002. In Der gesamtdeutsche Wähler. Stabilität und Wandel des Wählerverhaltens im wiedervereinigten Deutschland, Hrsg. Hans Rattinger, Oscar W. Gabriel, und Jürgen W. Falter, 235-249. Baden-Baden: Nomos.

Klein, Markus, und Manuela Pötschke. 2000. Wähler im sozialen Kontext. Mehrebenenanalysen des Wählerverhaltens bei den Bundestagswahlen der Jahre 1969 bis 1998. In 50 Jahre Empirische Wahlforschung in Deutschland. Entwicklung, Befunde, Perspektiven, Daten, Hrsg. Markus Klein, Wolfgang Jagodzinski, Ekkehard Mochmann und Dieter Ohr, 182-211. Wiesbaden: Westdeutscher Verlag.

Knack, Stephen, und Martha E. Kropf. 1998. For Shame! The Effect of Community Cooperative Context on the Probability of Voting. Political Psychology 19: 585-599. doi: 10.1111/0162-895X.00119.

Knutsen, Oddbjorn. 2004. Religious Denomination and Party Choice in Western Europe: A Comparative Longitudinal Study from Eight Countries, 1970-97. International Political Science Review 25: 97-128. doi: 10.1177/0192512104038169. 
Kotler-Berkowitz, Lawrence A. 2001. Religion and Voting Behaviour in Great Britain: A Reassessment. British Journal of Political Science 31: 523-554. doi: 10.1017/ S0007123401000205.

Lachat, Romain. 2007. A Heterogeneous Electorate. Political Sophistication, Predisposition Strength, and the Voting Decision Process. Baden-Baden: Nomos.

Layman, Geoffrey C., und John C. Green. 2006. Wars and Rumours of War: The Contexts of Cultural Conflict in American Political Behaviour. British Journal of Political Science 36: 61-89. doi: 10.1017/S0007123406000044.

Lenski, Gerhard. 1961. The Religious Factor: A Sociological Study of Religion's Impact on Politics, Economics, and Family Life. Garden City: Doubleday.

Levine, Jeffrey. 2005. Choosing Alone? The Social Network Basis of Modern Political Choice. In The Social Logic of Politics. Personal Networks as Contexts for Political Behavior, Hrsg. Alan S. Zuckerman, 132-151. Philadelphia: Temple University Press.

Liedhegener, Antonius. 2006. Macht, Moral und Mebrheiten. Der politische Katholizismus in der Bundesrepublik Deutschland und den USA seit 1960. Baden-Baden: Nomos.

McClurg, Scott D. 2003. Social Networks and Political Participation: The Role of Social Interaction in Explaining Political Participation. Political Research Quarterly 56: 449464. doi: $10.2307 / 3219806$.

Mead, George H. 1973. Geist, Identität und Gesellschaft. Frankfurt: Suhrkamp.

Minkenberg, Michael. 2010. Party politics, religion and elections in Western democracies. Comparative European Politics 8: 385-414. doi: 10.1057/cep.2009.5.

Mutz, Diana C. 2002a. Cross-Cutting Social Networks: Testing Democratic Theory in Practice. American Political Science Review 96: 111-126. doi: 10.1017/ S0003055402004264.

Mutz, Diana C. 2002b. The Consequences of Cross-Cutting Networks for Political Participation. American Journal of Political Science 46: 838-855. doi: 10.2307/3088437.

Olson, Laura R. 2012. Religion and American Public Life. Perspectives on Politics 10, 103106. doi: $10.1017 /$ S1537592711004348.

Pappi, Franz Urban, und Jens Brandenburg. 2010. Sozialstrukturelle Interessenlagen und Parteipräferenz in Deutschland. Stabilität und Wandel seit 1980. Kölner Zeitschrift für Soziologie und Sozialpsychologie 62: 459-483. doi: 10.1007/s11577-010-0111-4.

Pickel, Gert. 2010. Säkularisierung, Individualisierung oder Marktmodell? Religiosität und ihre Erklärungsfaktoren im europäischen Vergleich. Kölner Zeitschrift für Soziologie und Sozialpsychologie 62: 219-245. doi: 10.1007/s11577-010-0102-5.

Pickery, Jan. 2002. Contextual effects on the vote in Germany. A multilevel analysis. http:// www.econstor.eu/dspace/handle/10419/48987. Zugegriffen: 24.07.2012.

Przeworski, Adam, und Glaucio A. D. Soares. 1971. Theories in Search of a Curve: A Contextul Interpretation of Left Vote. American Political Science Review 65: 51-68. doi: 10.2307/1955043.

Raymond, Christopher. 2011. The continued salience of religious voting in the United States, Germany, and Great Britain. Electoral Studies 30: 125-135. doi: 10.1016/ j.electstud.2010.10.001.

Roßteutscher, Sigrid. 2007. CDU-Wahl 2005: Katholiken, Kirchgänger und eine protestantische Spitzenkandidatin aus dem Osten. In Die Bundestagswabl 2005. Analysen des Wahlkampfes und der Wahlergebnisse, Hrsg. Frank Brettschneider, Oskar Niedermayer und Bernhard Weßels, 321-347. Wiesbaden: VS Verlag für Sozialwissenschaften. 
Roßteutscher, Sigrid. 2012. Die konfessionell-religiöse Konfliktlinie zwischen Säkularisierung und Mobilisierung. Politische Vierteljahresschrift, Sonderheft 45: 111-133.

Schmitt-Beck, Rüdiger, Julia Partheymüller und Thorsten Faas. 2012. Einflüsse politischer Gesprächspartner auf Parteipräferenzen: Zur „sozialen Logik“ des politischen Verhaltens bei der Bundestagswahl 2009. Politische Vierteljahresschrift, Sonderheft 45: 465-488.

Schoen, Harald. 2003. Gleich und gleich gesellt sich gern - auch im Wahlkampf? Zur politischen Prägkraft der Sozialstruktur in Abhängigkeit zur Nähe zum Wahltag. Kölner Zeitschrift für Soziologie und Sozialpsychologie 55: 299-320. doi: 10.1007/s11577003-0052-2.

Schoen, Harald. 2009. Wahlsoziologie. In Politische Soziologie. Ein Studienbuch, Hrsg. Viktoria Kaina und Andrea Römmele, 181-208. Wiesbaden: VS Verlag für Sozialwissenschaften.

Schoen, Harald. 2011. Does ticket-splitting decrease the accuracy of recalled previous voting? Evidence from three German panel surveys. Electoral Studies 30: 358-365. doi: 10.1016/j.electstud.2011.01.007.

Stark, Rodney, und William S. Bainbridge. 1996. Religion, Deviance and Social Control. New York: Routledge.

Stark, Rodney, und Roger Finke. 2000. Acts of Faith. Explaining the Human Side of Religion. Berkley: University of California Press.

Statistische Ämter des Bundes und der Länder. 2012. Regionaldatenbank Deutschland. online: https://www.regionalstatistik.de/genesis/online/logon.

Tingsten, Herbert. [1937] 1975. Political Behavior. Studies in Election Statistics. New York: Arno Press.

Traunmüller, Richard. 2011. Moral Communities? Religion as a Source of Social Trust in a Multilevel Analysis of 97 German Regions. European Sociological Review 27: 346363. doi: 10.1093/esr/jcq011.

Traunmüller, Richard. 2012. Religion und Sozialkapital. Ein doppelter Kulturvergleich. Wiesbaden: Springer VS.

Traunmüller, Richard. 2013. The Dynamic Effect of Social Context on Political Preferences in Germany. Beitrag präsentiert auf der $71^{\text {th }}$ MPSA Annual Conference, 11.-13. April 2013, Chicago, IL.

van der Brug, Wouter, Sara B. Hobolt, und Claes H. de Vreese. 2009. Religion and Party Choice in Europe. West European Politics 32: 1266-1283. doi: 10.1080/ 01402380903230694.

van Deth, Jan, und Markus Tausendpfund (Hrsg.). 2013. Politik im Kontext: Ist alle Politik lokale Politik? Wiesbaden: Springer VS.

Verba, Sidney, Kay L. Schlozman, und Nancy Burns. 2005. Family Ties. Understanding the Intergenerational Transmission of Political Participation. In The Social Logic of Politics. Personal Networks as Contexts for Political Behavior, Hrsg. Alan S. Zuckerman, 95-114. Philadelphia: Temple University Press.

Weßels, Bernhard. 2011. Das Wahlverhalten sozialer Gruppen. In Zwischen Langeweile und Extremen. Die Bundestagswahl 2009, Hrsg. Hans Rattinger, Sigrid Roßteutscher, Rüdiger Schmitt-Beck und Bernhard Weßels, 103-117. Baden-Baden: Nomos.

Wolf, Christof. 1996. Konfessionelle versus religiöse Konfliktlinie in der deutschen Wählerschaft. Politische Vierteljahresschrift 37: 713-734. 
Zuckerman, Alan. 2007. The social logic of political choice: Picking a political party in the context of immediate social circles. Politische Vierteljahresschrift 48: 633-649. doi: 10.1007/s11615-007-0125-3.

Zuckerman, Alan S, Josip Dasovic, und Jennifer Fitzgerald. 2007. Partisan Families: the Social Logic of Bounded Partisanship in Germany and Britain. Cambridge: Cambridge University Press.

\section{Autorenangaben}

Kathrin Ackermann, M. A.,

Universität Bern, Institut für Politikwissenschaft,

Fabrikstrasse 8,

3012 Bern

kathrin.ackermann@ipw.unibe.ch

Dr. Richard Traunmüller,

University of Essex, Department of Government,

Wivenhoe Park,

Colchester CO4 3SQ

r.traun@essex.ac.uk 


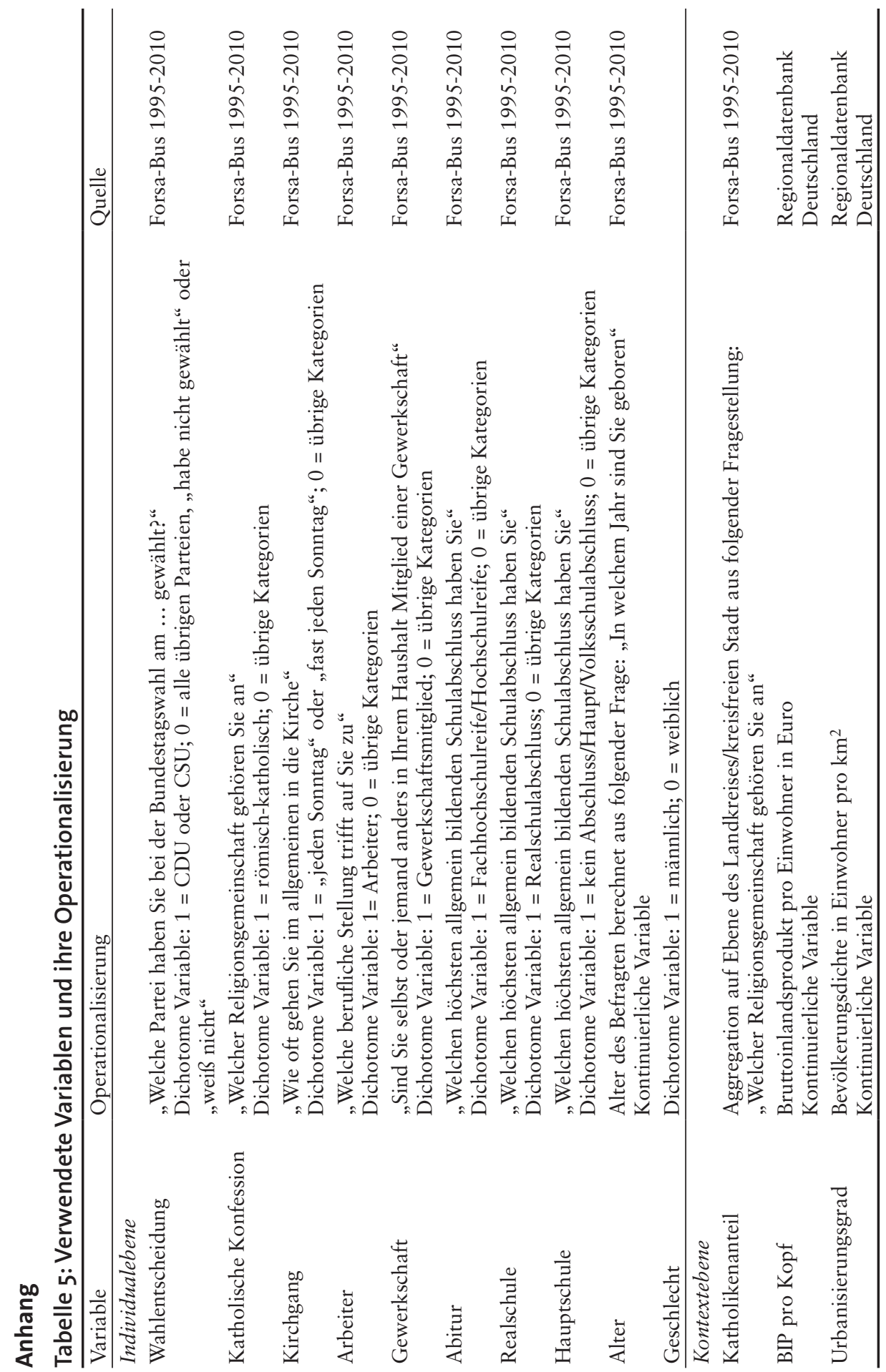




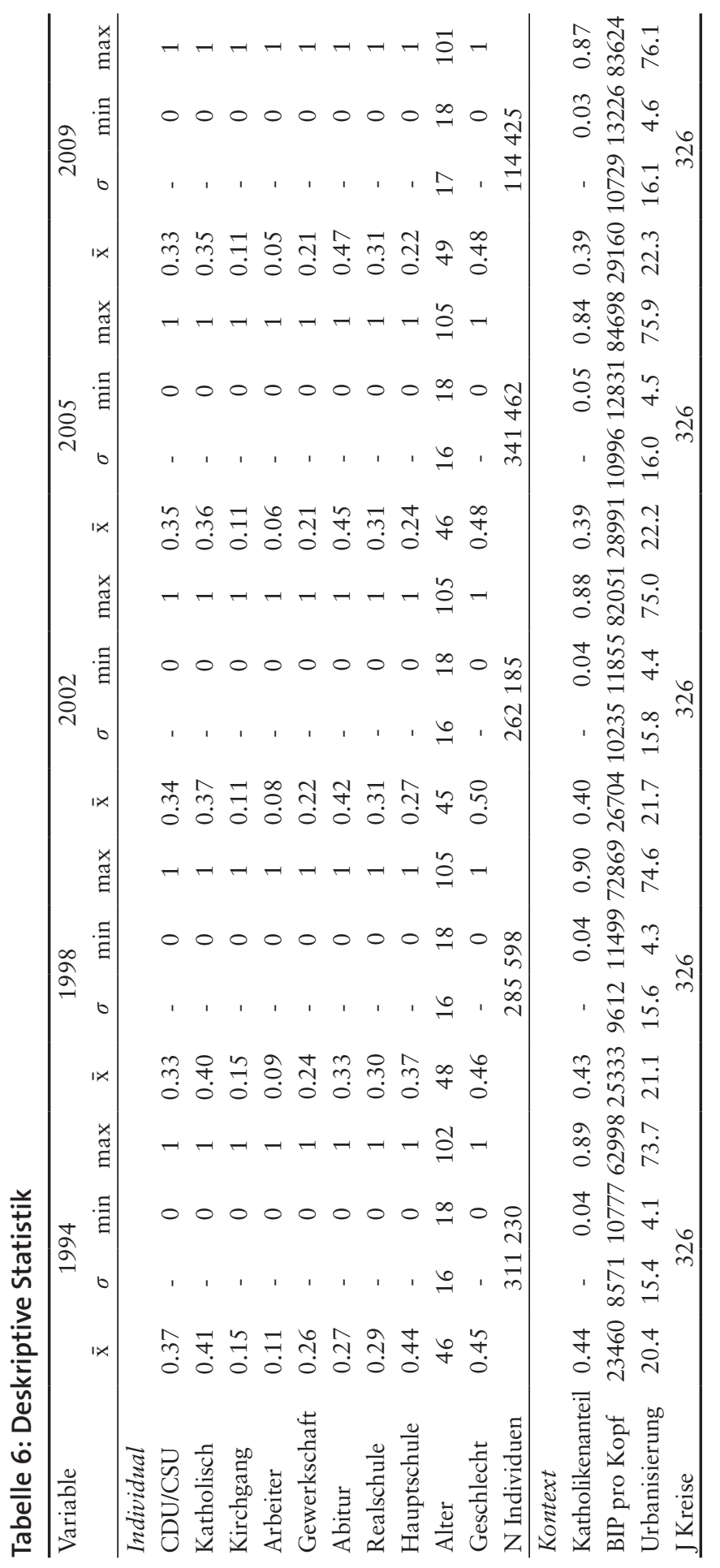


Tabelle 7: Modellvergleich für zwei verschiedene Spezifikationen des Kontexteffekts mit Kontrollvariablen

\begin{tabular}{lllllr}
\hline & & \multicolumn{2}{c}{ LR-Test } \\
\hline Bundestagswabl & Modell & Devianz & DF & $\chi^{2}(D F)$ & $\operatorname{Pr}\left(>\chi^{2}\right)$ \\
\hline \multirow{2}{*}{1994} & Linear & 373842.3 & 18 & & \\
& Quadratisch & 373826.6 & 20 & $15.662(2)$ & 0.000 \\
2002 & Linear & 326613.4 & 18 & & \\
& Quadratisch & 325602.3 & 20 & $11.133(2)$ & 0.004 \\
2005 & Linear & 306794.0 & 18 & & \\
& Quadratisch & 306786.4 & 20 & $7.6178(2)$ & 0.022 \\
2009 & Linear & 410260.0 & 18 & & \\
& Quadratisch & 410251.1 & 20 & $8.8602(2)$ & 0.012 \\
& Linear & 134733.7 & 18 & & \\
& Quadratisch & 134728.2 & 20 & $5.4817(2)$ & 0.065 \\
\hline
\end{tabular}

Anmerkung: Modelle aus Tabelle 4

Tabelle 8: Modellvergleich für zwei verschiedene Spezifikationen des Kontexteffekts - ohne Bayern

\begin{tabular}{llllll}
\hline & & \multicolumn{3}{c}{ LR-Test } \\
\hline Bundestagswabl & Modell & Devianz & DF & $\chi^{2}(\mathrm{DF})$ & $\operatorname{Pr}\left(>\chi^{2}\right)$ \\
\hline \multirow{2}{*}{1994} & Linear & 324215.4 & 7 & & \\
& Quadratisch & 324197.7 & 9 & $17.691(2)$ & 0.000 \\
2002 & Linear & 282041.0 & 7 & & \\
& Quadratisch & 282025.8 & 9 & $15.194(2)$ & 0.000 \\
2005 & Linear & 259206.3 & 7 & & \\
& Quadratisch & 259197.1 & 9 & \multirow{2}{*}{$9.202(2)$} & 0.010 \\
2009 & Linear & 347492.9 & 7 & & \\
& Quadratisch & 347485.7 & 9 & $7.184(2)$ & 0.028 \\
& Linear & 113805.4 & 7 & & \\
& Quadratisch & 113790.5 & 9 & $14.934(2)$ & 0.000 \\
\hline
\end{tabular}

Anmerkung: Modelle aus Tabelle 4 
Tabelle 9: Modellvergleich für zwei verschiedene Spezifikationen des Kontexteffekts - Logarithmische Modellierung

\begin{tabular}{lllllr}
\hline & & & \multicolumn{2}{c}{ LR-Test } \\
\hline Bundestagswabl & Modell & Devianz & DF & $\chi^{2}(\mathrm{DF})$ & $\operatorname{Pr}\left(>\chi^{2}\right)$ \\
\hline 1994 & Logarithmisch & 401083.0 & 7 & & \\
\multirow{2}{*}{1998} & Quadratisch & 401051.5 & 9 & $31.445(2)$ & 0.000 \\
& Logarithmisch & 351445.6 & 7 & & \\
2002 & Quadratisch & 351418.2 & 9 & $27.468(2)$ & 0.000 \\
2005 & Logarithmisch & 324281.4 & 7 & & \\
& Quadratisch & 324237.7 & 9 & $43.752(2)$ & 0.000 \\
2009 & Logarithmisch & 431851.6 & 7 & & \\
& Quadratisch & 431828.4 & 9 & $23.193(2)$ & 0.000 \\
& Logarithmisch & 141753.9 & 7 & & \\
& Quadratisch & 141738.4 & 9 & $15.481(2)$ & 0.000 \\
\hline
\end{tabular}

Anmerkung: Modelle aus Tabelle 4 